\title{
Finding Radial Network Configuration of Distribution System Based on Modified Symbiotic Organisms Search
}

\author{
Thuan Thanh Nguyen $\mathbb{D}^{1}{ }^{1}$ Thanh-Quyen Ngo $\left.\mathbb{D}\right)^{1}$ Thanh Long Duong $\mathbb{D}^{1}{ }^{1}$ \\ and Thang Trung Nguyen $\mathbb{D}^{2}$ \\ ${ }^{1}$ Faculty of Electrical Engineering Technology, Industrial University of Ho Chi Minh City, Ho Chi Minh City, Vietnam \\ ${ }^{2}$ Power System Optimization Research Group, Faculty of Electrical and Electronics Engineering, Ton Duc Thang University, \\ Ho Chi Minh City, Vietnam
}

Correspondence should be addressed to Thang Trung Nguyen; nguyentrungthang@tdtu.edu.vn

Received 20 April 2020; Revised 22 January 2021; Accepted 30 January 2021; Published 9 February 2021

Academic Editor: Alex Alexandridis

Copyright (C) 2021 Thuan Thanh Nguyen et al. This is an open access article distributed under the Creative Commons Attribution License, which permits unrestricted use, distribution, and reproduction in any medium, provided the original work is properly cited.

\begin{abstract}
Network reconfiguration (NR) is one of the most effective methods to reduce line power loss in the distribution system, which causes higher losses than the other parts of the power system. This paper proposes a modified symbiotic organisms search (MSOS) algorithm to solve the NR problem. For the purpose of enhancing the effectiveness of MSOS, the mutualism and parasitism strategies of the original symbiotic organisms search (SOS) have been modified to create better new solutions. In the mutualism strategy, the so-far best solution is updated immediately as soon as new solutions are created. In the parasitism strategy, the update is only implemented for the first half of control variables, whereas another half still remains unchanged. The comparison results between MSOS and SOS on twenty-five benchmark functions and different scales of test distribution systems with 14, 33, 69, and 119 nodes show that the improvement level of MSOS over SOS is significant with higher success rates and better quality of gained solutions. Similarly, MSOS also reaches better results than other methods in the literature. Consequently, MSOS can be a favorable method for determining the most appropriate configurations for the distribution systems.
\end{abstract}

\section{Introduction}

In general, there are four parts in the power system, namely, the power generation system, the transmission system, the distribution system, and the load components. The transmission system carries electricity from the power generation system to substations, whereas the distribution system transfers energy from the substation to the consumer. Because the distribution system works at a much lower voltage than the transmission system, the total losses of the distribution system are usually higher than those of the transmission system [1]. Thus, finding solutions for power loss reduction in the distribution system is one of the most important missions in the operation of the distribution system. The distribution system usually has a closed structure, but it is operated radially due to some technical advantages of opened operation, compared to closed operation. Therefore, in general, there exist some switches with open status in the system to maintain the radial topology of the distribution system. As operation requirements are changed, such as fault isolation, maintenance, overload reduction, etc., these switches are changed to closed status while the other switches in the system are switched to open status. In addition to the above purposes, the status of the switches may be changed to transfer a part of the loads from a given branch to others. The process of changing the status of the switches on the distribution system will change the operating structure of the system. Thus, this process is called network reconfiguration. However, this is a complex problem with discrete and nonlinear features. Furthermore, the number of possible solutions for the problem is also very high, which can be up to $2^{n}$ structures for $n$ switches equipped on the system. Therefore, finding optimal solutions for the NR problem is a big challenge for researchers. 
Applying successfully the solving methods for the NR problem for power loss reduction will be a prerequisite for their application to the NR problem to reduce energy losses as well as satisfy other objective functions.

The NR problem was first proposed for power loss reduction and solved by the heuristic technique [2]. In the aforementioned work, in order to find the optimal configuration, all of initial switches are closed and then, switches are changed to open status in turn to form a radial configuration. Later on, some of these techniques are developed based on improving the above technique, such as methods in $[3,4]$, with the purpose of gaining a better solution or for easier implementation. But, in general, these methods face many limitations related to the quality of the obtained solution as well as the dependency on the type of objective functions. Therefore, up to the present, the development of methods has not really attracted much attention from researchers. Due to the advantages in the application of various objective functions and handling constraints, metaheuristic algorithms are widely used for problems related to power systems in general and the NR problems in particular. Both the well-known algorithms and recently developed algorithms have been applied to solve the reconfiguration problems, such as genetic algorithm (GA) [5-7], particle swarm optimization (PSO) [8-10], ant colony algorithm [11-13], cuckoo search algorithm $[14,15]$, adaptive shuffled frogs leaping algorithm (ASFLA) [16], runner root algorithm (RRA) [17, 18], improved adaptive imperialist competitive algorithm (IAICA) [19], fireworks algorithm (FWA) [20], and hybrid of wild goats and exchange market algorithms (WGEMA) [21]. For applying metaheuristic methods to the NR problem, it is noted that the effectiveness of an algorithm may be dependent on control parameters of the algorithm itself. The selection of suitable control parameters can bring a good solution to the problem, but the selection of inappropriate control parameter values may lead to bad solutions being obtained. Therefore, in this respect, algorithms with little or no control parameters have an initial advantage when they are used to solve NR problems. Furthermore, in fact, it is impossible to have a high-quality algorithm for all problems. An optimization algorithm may outperform the other algorithms for a given problem, but its results for another problem may be worse than those of the other algorithms [22]. Therefore, the demand for adding new solving methods to the NR problem is also a matter of concern.

Symbiotic organisms search (SOS) is developed based on the ideal of interaction among organisms in the ecosystem for surviving [23]. There are three mechanisms to generate new solutions in the SOS consisting of mutualism, commensalism, and parasitism mechanisms. In the mutualism mechanism, the considered solution and a random solution in the ecosystem are renewed, whereas in the commensalism mechanism, only the considered solution is updated based on the information of a random solution in the ecosystem. Finally, the considered solution is used to generate a new solution for replacing a random one in the ecosystem. Unlike GA, PSO, and the other methods, the SOS does not require additional control parameters during the searching process. This feature helps to increase the stability and simplicity of the SOS for finding the optimal solution. Therefore, the SOS has received much attention from researchers in other related fields. The effectiveness of the SOS compared to other methods for benchmark functions has been demonstrated in [23]. In addition, the SOS and its improved versions have been effectively applied for many technical problems, such as engineering structure problems $[24,25]$ and the resource leveling problem in the construction field [26], and vehicle routing and traveling salesman problems in the transport field $[27,28]$. In the field of power system, the problems consisting of distributed generation placement [29-33], capacitor placement [34], dispatch problem $[35,36]$, and NR problem [37] have been successful solved by SOS. In addition, there are a lot of problems related to energy optimization and engineering design that have been successful carried out by the SOS [38]. Based on the parameter-less feature of the SOS and its high ability to solve technical problems as well as problems in the field of electrical systems, the application of the SOS to the NR problem for power loss reduction promises to provide a very effective tool for finding the optimal solution to the NR problem for other targets.

In this paper, SOS is adapted for the NR problem. However, in order to increase the efficiency of searching for optimal solutions, we propose a modified version of SOS (MSOS) for the NR problem. For the MSOS, the exploitation search strategy of the original SOS is modified to create new solutions that are not too far from the considered solution, and the so-far best solution is updated as soon as new solutions are generated in the ecosystem. In order to evaluate the performance of the proposed MSOS method for the NR problem, it is used to find the optimal network configurations for minimizing power loss via four distribution systems comprising 16, 33, 69, and 119 nodes systems. The simulation results of the MSOS have been compared to those of the SOS and the other algorithms in the literature. The main contributions of the paper can be stated as follows:

(i) Propose a modification for the exploited search strategy to generate new solutions around the considered solutions and a modification for the explored search strategy to get better information for generating new solutions

(ii) The MSOS is adapted to solve the NR problem for power loss reduction

(iii) The MSOS can reach a higher success rate and better solution quality than the original SOS for all test distribution systems

Other remaining parts of the paper are as follows: Mathematical formulation of the NR problem is shown in Section 2. The modified symbiotic organisms search is presented in Section 3. The application of the MSOS for the NR problem is explained in Section 4 . The numerical results are given in Section 5. Finally, conclusions are presented in Section 6. 


\section{Mathematical Formulation for the Network Reconfiguration Problem}

The NR problem can be considered as a nonlinear optimization problem in which a solution for the NR problem is selected from all possible radial configurations. The best solution is a radial configuration that causes the smallest power loss and satisfies a set of constraints of the NR problem. The mathematical functions of the NR problem can be observed as follows:

$$
\min \text { obj }=\sum_{i=1}^{N_{\mathrm{br}}} \Delta P_{i},
$$

where obj is the objective function of the NR problem; $\Delta P_{i}$ is the power loss of the ith branch of the distribution system; and $N_{b r}$ is the number of branches existing in the distribution system.

The obtained solution must subject to the below constraints:

The radial topology constraint: each candidate of network configuration must be a radial topology. If the topology of the distribution system is radial, the below equation will be satisfied [39]:

$$
|\operatorname{det}(A)|=1
$$

where $\mathrm{A}$ is a connection matrix of the distribution system with $N_{\text {br }}$ rows and $N_{\text {bus }}$ columns. $A(i, j)$ will be equal to 0 , if the ith branch is not connected from/to the $j^{\text {th }}$ node. Otherwise, $A(i, j)$ will be equal $1 /-1$, if the $i^{\text {th }}$ branch is connected from/to the $j^{\text {th }}$ node. $N_{\text {bus }}$ is the number of nodes in the distribution system.

The voltage magnitude and current constraints are as follows:

$$
\begin{gathered}
V_{\text {low }} \leq V_{i} \leq V_{\text {up }} ; \quad \forall i \in N_{\text {bus }} \\
I_{i} \leq I_{\text {rate }, i} ; \quad \forall i \in N_{\text {br }}
\end{gathered}
$$

where $V_{\text {low }}$ and $V_{\text {up }}$ are, respectively, the lower and upper limits of voltage magnitude of each node. $V_{i}$ is the voltage magnitude of the ith node. $I_{\text {rate }, i}$ and $I_{i}$ are the rated current and operating current of the ith branch, respectively. $N_{b r}$ is the number of branches in the distribution system.

\section{The Modified Symbiotic Organisms Search}

3.1. The Original Symbiotic Organisms Search. In the SOS, each organism in the ecosystem is considered as a solution of the problem. The initial ecosystem can be performed by using the following popular model:

$$
O_{i}=O_{\min }+\mu_{1} \cdot\left(O_{\max }-O_{\min }\right) ; \quad i=1,2, \ldots, N,
$$

where

$$
\begin{array}{ll}
O_{\min }=\left[x_{j, \min }\right] ; & j=1,2, \ldots, D, \\
O_{\max }=\left[x_{j, \max }\right] ; & j=1,2, \ldots, D,
\end{array}
$$

where $O_{i}$ is the $i^{\text {th }}$ organism in the ecosystem; $O_{\max }$ and $O_{\min }$ represent the upper and lower bounds of the organism, respectively; $x_{j, \min }$ and $x_{j, \max }$ are the lowest and the highest values of the $j^{\text {th }}$ control variable, respectively; $\mu_{1}$ is a random number in $[0,1] ; N$ is ecosystem size; and $D$ is the number of control variables of the problem.

Then, the quality of each organism is determined by calculating the adaptive function (fit) and the best organism $\left(O_{\text {best }}\right)$, with the best adaptive function value being selected.

In order to explore the search space, the SOS uses the information of a random organism $\left(O_{r}\right)$ in the ecosystem and the best organism to produce a new organism for the $i^{\text {th }}$ one. In this step, the randomly selected organism is also updated. Due to generating both the new organisms, the exploration strategy is also called mutualism strategy. The solutions are updated by the following rule:

$$
\left\{\begin{array}{l}
O_{i, \text { new }}=O_{i}+\mu_{2} \cdot\left(O_{\text {best }}-M_{\mathrm{vec}} \cdot \mathrm{BF}_{1}\right), \\
O_{r, \text { new }}=O_{r}+\mu_{3} \cdot\left(O_{\text {best }}-M_{\text {vec }} \cdot \mathrm{BF}_{2}\right),
\end{array}\right.
$$

where $\mathrm{BF}_{1}$ and $\mathrm{BF}_{2}$ are benefit factors selected in range of $[1,2]$. They reflect the degree of the obtained benefit of the organisms for interacting with each other. $\mu_{2}$ and $\mu_{3}$ are random numbers in $[0,1] . \mathrm{M}_{\mathrm{vec}}$ is a mean solution of the $i^{\text {th }}$ and $j^{\text {th }}$ organisms determined by the following equation:

$$
M_{\mathrm{vec}}=\frac{O_{i}+O_{r}}{2}
$$

It is not sure whether each variable of new organisms exactly satisfies the lower and the upper limits. Therefore, each new variable of the new organisms must be checked and corrected as follows:

$$
x_{j \text {, new }}= \begin{cases}x_{j, \min } ; & \text { if } x_{j, \text { new }}<x_{j, \text { min }}, \\ x_{j, \text { max }} ; & \text { if } x_{j, \text { new }}>x_{j, \text { max }}, \\ x_{j}, \\ j=1, \ldots, D\end{cases}
$$

Then, the selection technique is used to retain more effective organisms and abandon ineffective organisms. The quality of the new organisms is evaluated by calculating the adaptive function. Then, the current organisms will be replaced with such new ones if the adaptive function of the new organisms is better than that of the current ones. This selection is described as follows:

$$
\begin{aligned}
& O_{k}=\left\{\begin{array}{ll}
O_{k, \text { new }} ; & \text { if } \text { fit }_{k, \text { new }}<\text { fit }_{k}, \\
O_{k} ; & \text { else, }
\end{array} \quad k=i, k=r\right. \\
& \mathrm{fit}_{k}= \begin{cases}\mathrm{fit}_{k, \text { new }} ; & \text { if } \mathrm{fit}_{k, \text { new }}<\mathrm{fit}_{k}, \quad k=i, k=r . \\
\mathrm{fit}_{k} ; & \text { else, }\end{cases}
\end{aligned}
$$

Unlike the mutualism strategy, only the ith organism is updated once again in the commensalism strategy. In this strategy, a random organism is also chosen to interact with the $i$ th one as follows: 


$$
O_{i, \text { new }}=O_{i}+\mu_{4} \cdot\left(O_{\text {best }}-O_{j}\right) \text {, }
$$

where $\mu_{4}$ is a random number in the range $[-1,1] ; O_{j}$ is the organism selected randomly from the ecosystem.

The new organism's fitness function value is calculated. Then, the $i$ th organism is updated once again by using the following selection technique:

$$
\begin{aligned}
O_{i} & = \begin{cases}O_{i, \text { new }}, & \text { if fit } \\
O_{i, \text { new }}, & \text { else, }\end{cases} \\
\text { fit }_{i} & = \begin{cases}\text { fit }_{i, \text { new }}, & \text { if fit } \\
\text { fit }_{i}, & \text { elsew }\end{cases}
\end{aligned}
$$

In order to exploit the search space, the SOS produces a new solution around the current solution through the parasitism strategy. This strategy is like the mutation of the genetic algorithm. However, the number of control variables of the current solution selected for changing the value is a random number. In this strategy, a new organism is produced by modifying some control variables of the selected organism. To select variables, a vector $\left(X_{\text {perm }}\right)$ is used to contain the random permutation of 1 to $D$. Then, the number of control variables selected for modification is determined as follows:

$$
\text { pick }=X_{\text {perm }}\left(1: \operatorname{round}\left(\mu_{5} \cdot D\right)\right),
$$

where pick is a vector containing the position of variables in the organism; $\mu_{5}$ is a random number in range $[0,1]$; and round () is the round function.

The new organism $\left(O_{\mathrm{par}}\right)$ is generated by modifying control variables of the $i$ th organism as follows:

$$
x_{j, \text { new }}=\left\{\begin{array}{ll}
x_{\text {par }, j} ; & \text { if } j \in \text { pick, } \\
x_{i, j} ; & \text { else, }
\end{array} \quad j=1, \ldots, D,\right.
$$

where $x_{p a r, j}$ is a random variable generated within its lower and upper limits.

The new organism's quality is determined by finding the fitness function value. However, the new organism is not used to update the ith organism; instead, it is used to replace a random organism selected in the ecosystem as follows:

$$
\begin{aligned}
& O_{j}= \begin{cases}O_{\text {par }} ; & \text { if fit } \\
O_{j} ; & \text { else, }\end{cases} \\
& \text { fit }_{j}= \begin{cases}\text { fit }_{\text {par }}, & \text { if fit } \\
\text { fit }_{j}, & \text { else, }\end{cases}
\end{aligned}
$$

where $O_{j}$ is the organism selected randomly from the ecosystem.

After performing three strategies for the $i^{\text {th }}$ organism in the ecosystem, the ith organism is renewed two times. The first renewal is performed in the mutualism strategy and the second renewal is executed in the commensalism strategy. In addition, the information of the ith organism also helps to renew two other organisms in the ecosystem. The first one is obtained in the mutualism strategy and the second one is renewed in the parasitism strategy.

At the final step of the SOS, the best organism is updated based on the adaptive function of all organisms in the ecosystem.

The next organism continues to be renewed by the mutualism, commensalism, and parasitism strategies of the SOS until the final organism of the ecosystem is assessed. The ecosystem updating process is performed until the number of adaptive function evaluations reaches the maximum value (MFE). The whole computation process of the SOS is expressed in Figure 1.

3.2. The Modified SOS. In the mutualism strategy of the SOS, two organisms $O_{i}$ and $O_{r}$ interact with each other to create new organisms. In addition, these two organisms also interact with the best so-far organism in the population. In this case, the best so-far organism is like the reference point in the search space for guiding other organisms. However, thanks to the interaction vector between two organisms, the mutualism strategy still maintains its exploration capability. Therefore, the mutualism strategy helps the SOS to ensure the balance between exploration and exploitation. In the commensalism strategy, only organism $O_{i}$ is updated, whereas the organism that is selected for interacting with the organism $O_{i}$ is not affected. In addition, the organism $O_{i}$ also interacts with the best so-far organism. It is worth noting that the organism tends to move toward the best so-far organism when the difference between the best so-far organism and $O_{j}$ organism is very tiny. Therefore, this strategy ensures the best exploitation of the space around the best sofar organism. It is noted that in the commensalism strategy, the best so-far organism continues to be used to create the new organism. However, in the original SOS, the best so-far organism is not updated after performing the mutualism strategy, although there are two new organisms created in the mutualism strategy. Thus, in order to make sure that the best so-far organism used in the commensalism strategy is the best one of the current population, the best so-far organism needs to be updated again. For the parasitism strategy, the parasitic vector is generated by a combination of random variables and variables of the organism $O_{i}$. Therefore, this strategy mainly enhances the exploitation ability of the SOS by creating an organism around the current organism. In addition, the random variables in this strategy allow the SOS to travel to both nonvisited and visited spaces [25]. Therefore, this strategy also helps the SOS to avoid being trapped in the local optima and to get the diversity of organisms of the population. However, due to a high number of generated random variables, it can be seen that the exploitation ability of parasitism strategy is considered to be weaker than its exploration capacity.

Based on the aforementioned characteristics, this work will focus on improving the effectiveness of the SOS for the NR problem by making two adjustments to the original SOS. Firstly, in order to ensure that the best organism is used in 


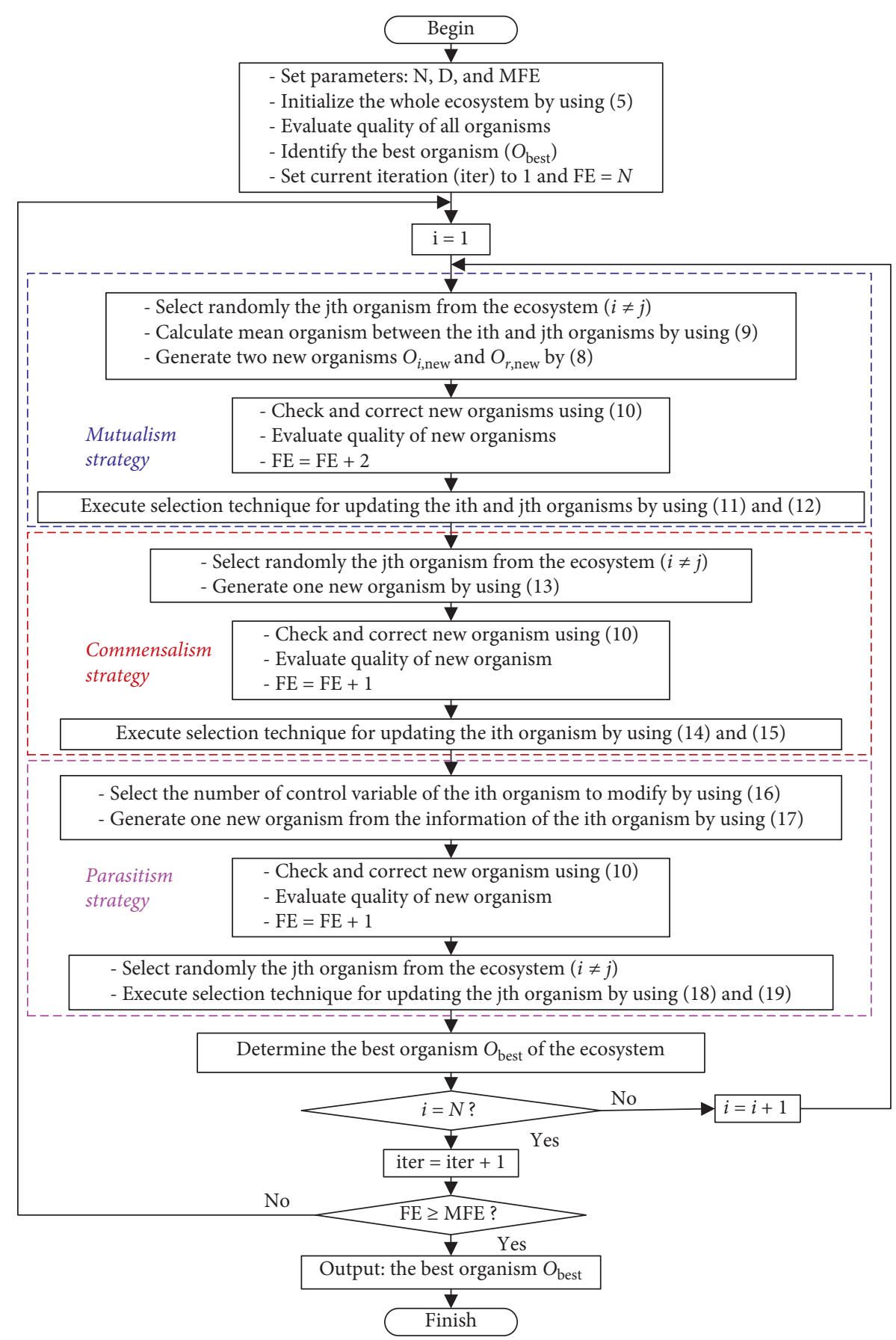

FIgURE 1: The flowchart of using the SOS for solving a typical optimization problem.

the commensalism strategy, the best so-far organism should be updated immediately after implementing the mutualism strategy. Secondly, the parasitism strategy is adjusted to improve the exploitation capacity of the SOS. Details of the adjustments are presented as follows:

3.2.1. The First Modification. The mutualism, commensalism, and parasitism strategies of the SOS create four new organisms. For the mutualism and commensalism strategies, the considered organism will interact with the best so-far organism to create three new organisms, with two organisms of the mutualism strategy and another one of the commensalism strategy. The interaction with the best so-far organism enables created new organisms to follow the best so-far organism. However, two new organisms have been generated and evaluated in the mutualism strategy. The new organism's fitness value may be better than that of the best organism. Thus, it is necessary to update the best organism again before moving to the commensalism strategy. 


$$
O_{\text {best }}= \begin{cases}\text { temp_- } O_{\text {best }} ; & \text { if fit }\left(\text { temp_O } O_{\text {best }}\right)<\text { fit }\left(O_{\text {best }}\right), \\ O_{\text {best }} ; & \text { else, }\end{cases}
$$

where temp_ $O_{\text {best }}$ is determined as follows:

$$
\text { temp_O } O_{\text {best }}= \begin{cases}O_{i} ; & \text { if fit }\left(O_{i}\right)<\operatorname{fit}\left(O_{r}\right), \\ O_{r} ; & \text { else. }\end{cases}
$$

3.2.2. The Second Modification. In the parasitism strategy, the $i^{\text {th }}$ organism is also chosen to become the parasite. The parasite is generated by a combination of random variables and variables of the organism $O_{i}$. Then, the number of control variables of the parasite from one to the length of solution vector is selected randomly. This strategy helps the SOS to search around the current solution for finding a better solution compared to another current solution. However, if the number of the selected control variables is too high, the newly produced solution will be completely different from the current, and it will not be an effective one. In this case, a small number of random variables will ensure good exploitation of the space around $O_{i}$.

In addition, for the network reconfiguration, changing some of the open switches of a radial configuration may lead to another configuration. This configuration is definitely different from the original one because each radial configuration is created by the geometric structure that is formed by open switches. Therefore, to search around the current solution, the maximum number of control variables of the chosen parasite for update is limited to a half of that in the original SOS. This modification helps to generate the new solution, which is located around the current solution. Therefore, equation (14) is replaced with the following equation:

$$
\text { Pick }=X_{\text {perm }}\left(1: \operatorname{round}\left(\mu_{5} \cdot \frac{D}{2}\right)\right) .
$$

The whole computation process of the proposed MSOS method is presented in Figure 2.

\section{Solving the Network Reconfiguration Using the Proposed MSOS Method}

4.1. Ecosystem Initialization. In order to perform the program of searching for an optimal solution, the MSOS generates a set of initial candidate solutions (called the ecosystem) by using (4). In (4), $O_{\min }$ and $O_{\max }$ are the minimum and maximum positions of open switches in the loop vector containing the switches. The switches in the loop vector are determined by the following steps:

Close the first initial open switch of the initial radial configuration. At that time, the distribution system will exist only as a close loop.

Select all switches in the close loop and store in the first loop vector.
Restore the first switch to the initial status and close the next initial open switch to determine the switches that belong to the next loop vector.

The process is repeated until the last loop vector is determined.

Thus, $x_{j, \min }$ is equal to 1 for all control variables and $x_{j, \max }$ is the length of the $j$ th loop vector. Each control variable of the organism will be mapped to the corresponding loop vector to take out the corresponding open switch. Therefore, it must be an integer value and all control variables are modified as follows:

$$
O_{i}=\operatorname{round}\left[O_{i}\right] ; \quad i=1,2, \ldots, N .
$$

After each solution is mapped to take out the set of open switches, the data of the distribution system is updated. In case the radial topology constraint is not satisfied, the corresponding adaptive function value is assigned to a bad value. On the contrary, if the radial topology constraint is satisfied, the load flow program is executed to calculate power loss, the minimum and maximum voltage magnitudes, and the maximum load-carrying factor of the radial configuration system. As a result, the adaptive function for evaluating the quality of the candidate network configuration, which involves two parts, consisting of the objective function and the constraints of the NR problem, can be constructed as follows:

$$
\begin{aligned}
\text { fit }= & \text { obj }+K \cdot\left[\left(\max \left(V_{\text {low }}-V_{\text {min }}\right), 0\right)\right. \\
& \left.+\left(\max \left(V_{\max }-V_{\text {up }}\right), 0\right)+\left(\max \left(\mathrm{LCF}_{\max }-1\right), 0\right)\right],
\end{aligned}
$$

where fit is the adaptive function value of each radial network configuration; $K$ is the penalty coefficient; and $V_{\text {min }}$ and $V_{\max }$ are the minimum and maximum voltage magnitudes of the distribution system. $\mathrm{LCF}_{\max }$ is the maximum load-carrying factor of the distribution system.

4.2. Processes of Newly Updated Solutions. In the MSOS, each organism in the ecosystem is updated two times. In addition, its information is also used to update two other organisms in the ecosystem.

In the mutualism strategy, from the current organism $O_{i}$ and the random organism selected $O_{r}$, two new solutions are generated as follows:

$$
\left\{\begin{array}{l}
O_{i, \text { new }}=\operatorname{round}\left[O_{i}+\mu_{2} \cdot\left(O_{\text {best }}-M_{\mathrm{vec}} \cdot \mathrm{BF}_{1}\right)\right], \\
O_{r, \text { new }}=\operatorname{round}\left[O_{r}+\mu_{3} \cdot\left(O_{\text {best }}-M_{\mathrm{vec}} \cdot \mathrm{BF}_{2}\right)\right] .
\end{array}\right.
$$

Then, the correction is executed to handle the violation of each control variable. In more detail, the control variables will be assigned to the lower bound if they are less than the lower limit. They will be assigned to the upper bound if they are higher than the upper limit. The correction method can be implemented as follows: 


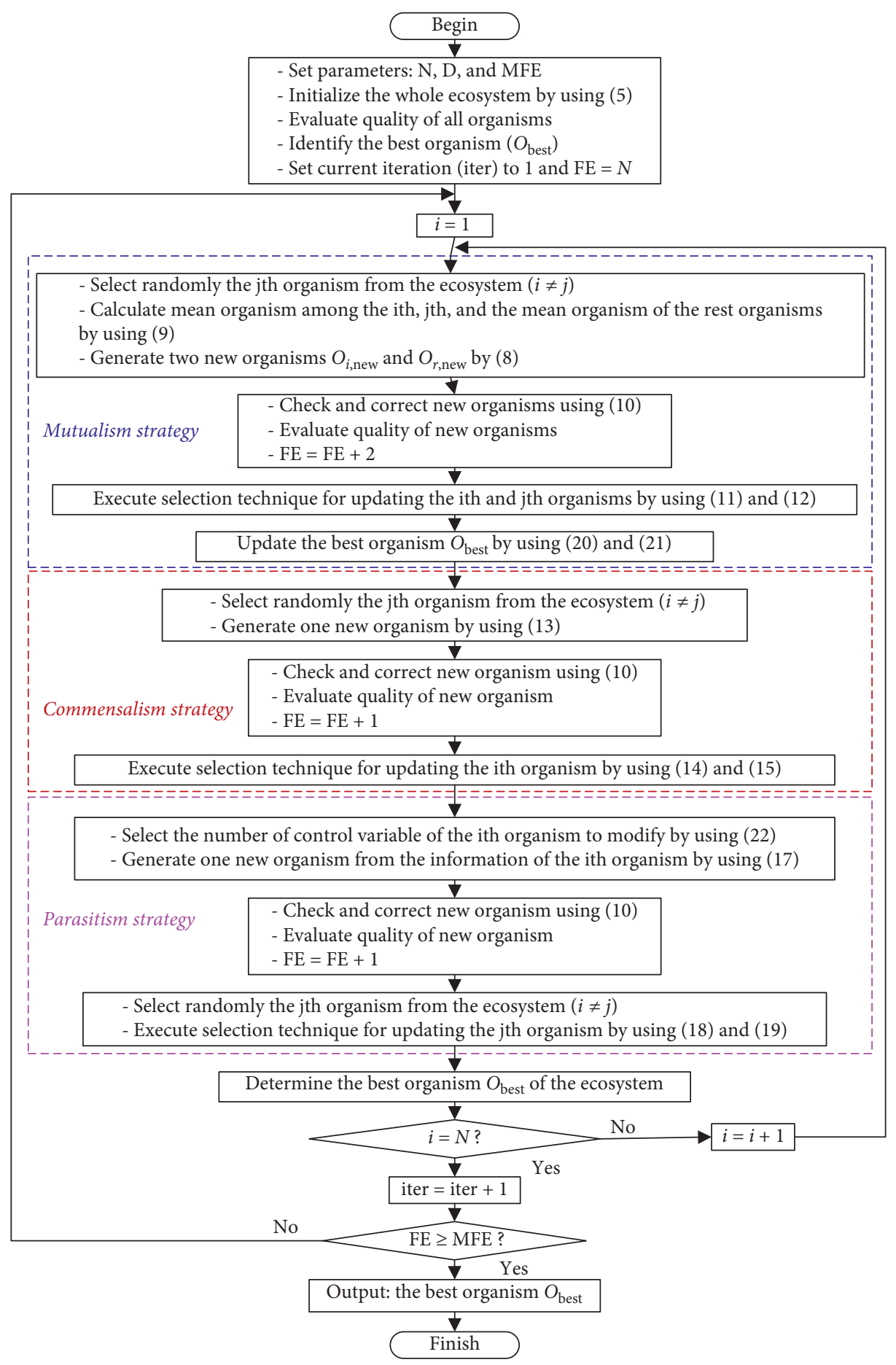

FigURE 2: The flowchart of using the MSOS for solving a typical optimization problem.

$$
x_{j, \text { new }}= \begin{cases}1 ; & \text { if } x_{j, \text { new }}<1, \\ \text { length }\left(\text { loop_vector }_{j}\right) ; & \text { if } x_{j, \text { new }}>\text { length }\left(\text { loop_vector }_{j}\right) ; \quad j=1, \ldots, D . \\ x_{j, \text { new }} ; & \text { otherwise },\end{cases}
$$

The quality of the new solutions is evaluated by using (22). Then, (9) and (10) are used for updating the two corresponding organisms.
As mentioned in the second modification, after updating the two new solutions using the mutualism strategy, the best so-far organism is updated by using (18) and (19). Then, a 
new solution is generated by using the commensalism strategy as follows:

$$
O_{i, \text { new }}=\operatorname{round}\left[O_{i}+\mu_{4} \cdot\left(O_{\text {best }}-O_{j}\right)\right] .
$$

Quality of the new solution obtained in the mutualism strategy is evaluated by using (22). Then, (12) and (13) are used for updating the current organism $O_{i}$.

After performing the mutualism and commensalism strategies, the parasitism strategy is executed to generate a new solution. In this strategy, the information of the current organism $O_{i}$ is also used to update another organism in the ecosystem. In order to generate the new solution, some variables of the current organism $O_{i}$ are selected to change values while the remaining variables are unchanged. The number of control variables for changing the value is selected by using (20). Then, their value is generated by using (15). In order to ensure the integer form for control variables, the new solution is modified by using the round function as follows:

$$
O_{\mathrm{par}}=\operatorname{round}\left[\mathrm{O}_{\mathrm{par}}\right] \text {. }
$$

The quality of new solution is evaluated by using (22). Then, (16) and (17) are used for updating a random organism selected in the ecosystem.

After performing mutualism, commensalism, and parasitism strategies for the current organism $O_{i}$, the best so-far organism is updated again before moving to the next organism in the ecosystem. This process is executed until the entire ecosystem is surveyed and updated.

4.3. Termination of Searching. It can be seen that when the last solution in the ecosystem is surveyed, the number of adaptive function evaluations is $4 \times N$ because there are $4 \times$ $N$ new solutions. Therefore, in this study, the stopping condition of the MSOS for the NR problem is determined based on the number of adaptive function evaluations. The ecosystem will be updated until the number of adaptive function evaluations reaches the preset maximum value (MFE). The iteration variable (iter) is used to count the number of times that the ecosystem is updated. At the last iteration, the radial configuration with the lowest adaptive function value is identified as the best solution for the run. To ensure the reliability of the solution obtained, the MSOS is run independently with a predetermined number of times $\left(N_{\text {runs }}\right)$. After finishing $N_{\text {runs }}$, a set of solutions consisting of $N_{\text {runs }}$ radial configurations will be obtained. Then, the best configuration is considered as the solution of the NR problem. In addition, the performance of the proposed MSOS method is also evaluated via the minimum, average, and maximum adaptive function values, and the standard deviation of the number of runs.

\section{Numerical Results}

5.1. Performance of the MSOS for Benchmark Problems. In order to show the efficiency of modifications of the MSOS, it is compared with the SOS for the 25 benchmark functions, which are taken from $[23,33,40]$ and presented in Table 1. The benchmark functions include 2 variables for the $1^{\text {st }}$ to the $11^{\text {th }}$ functions, 4 variables for the $12^{\text {th }}$ function, five variables for the $13^{\text {th }}$ function, 10 variables for the $14^{\text {th }}$ to $15^{\text {th }}$ functions, and 30 variables for the $16^{\text {th }}$ to $25^{\text {th }}$ functions. The parameters of the SOS and the MSOS consisting of population size and maximum number of evaluations are chosen to be 30 and 100000 , respectively. Both of the methods are executed independently for 50 runs to obtain the maximum fitness (Max), minimum fitness (Best), mean fitness (Mean), standard deviation (STD), the success rate, and the mean convergence iterations (Mean con. iter.) for comparisons.

The obtained results for the 25 benchmark functions are shown in Tables 2 and 3. Both the MSOS and the SOS have determined the minimum values for all functions except for the $19^{\text {th }}$ and the $22^{\text {nd }}$ functions. For the $1^{\text {st }}, 2^{\text {nd }}, 3^{\text {rd }}, 4^{\text {th }}, 6^{\text {th }}$, $8^{\text {th }}, 9^{\text {th }}$, and $16^{\text {th }}$ mathematical functions, both the MSOS and the SOS have found the minimum value in each run, but the MSOS usually converges earlier than the SOS. Only the MSOS converges later than the SOS for the $7^{\text {th }}, 13^{\text {th }}, 21^{\text {th }}$, and $23^{\text {th }}$ functions. For the $10^{\text {th }}, 14^{\text {th }}, 17^{\text {th }}, 18^{\text {th }}, 20^{\text {th }}, 24^{\text {th }}$, and $25^{\text {th }}$ functions, the performance of the MSOS and the SOS are similar, but meanwhile the success rate, standard deviation of the fitness function, and the mean number of convergence iterations of the MSOS are better than those of the SOS for the $11^{\text {th }}$ function. Similarly, for the $12^{\text {th }}$ function, the mean value and the standard deviation of the fitness function and the mean number of convergence iterations gained by the MSOS are better than those of the SOS. For the $19^{\text {th }}$ function, the mean value of the fitness function and the mean number of the convergence iterations of the MSOS are better than those of the SOS. Meanwhile, the performance of the SOS is better than the MSOS in terms of the mean value of the fitness function for the $5^{\text {th }}$ and $15^{\text {th }}$ functions. For the $22^{\text {nd }}$ function, the SOS has the better performance than the MSOS, but both of the methods have not determined the minimum value for this function.

5.2. Performance of the MSOS for the NR Problem. In this section, the effectiveness of the proposed MSOS is evaluated on four test distribution systems comprising 14, 33, 69, and 119 -node systems. The obtained results by the MSOS are compared with those of the original SOS. Both the MSOS and the SOS are implemented on Matlab software and run on a personal computer with a processor of Core i5 $2.4 \mathrm{GHz}$ and $4 \mathrm{~GB}$ of RAM. The control parameters of the two methods for the test systems are set as given in Table 4 .

5.2.1. The 14-Node Test System. The $23 \mathrm{kV}, 14$-node test system contains 3 feeders and 13 load nodes. Three initial open switches are $\{s 15, s 16$, and $s 17\}$, which cause a power loss of $511.4356 \mathrm{~kW}$. The system data are referenced from [5]. The single diagram of the system for the initial configuration is given in Figure 3.

The simulation results for the 14-node system are presented in Table 5. From the table, the total power loss of the system has been reduced from $511.4356 \mathrm{~kW}$ to $466.1267 \mathrm{~kW}$ 


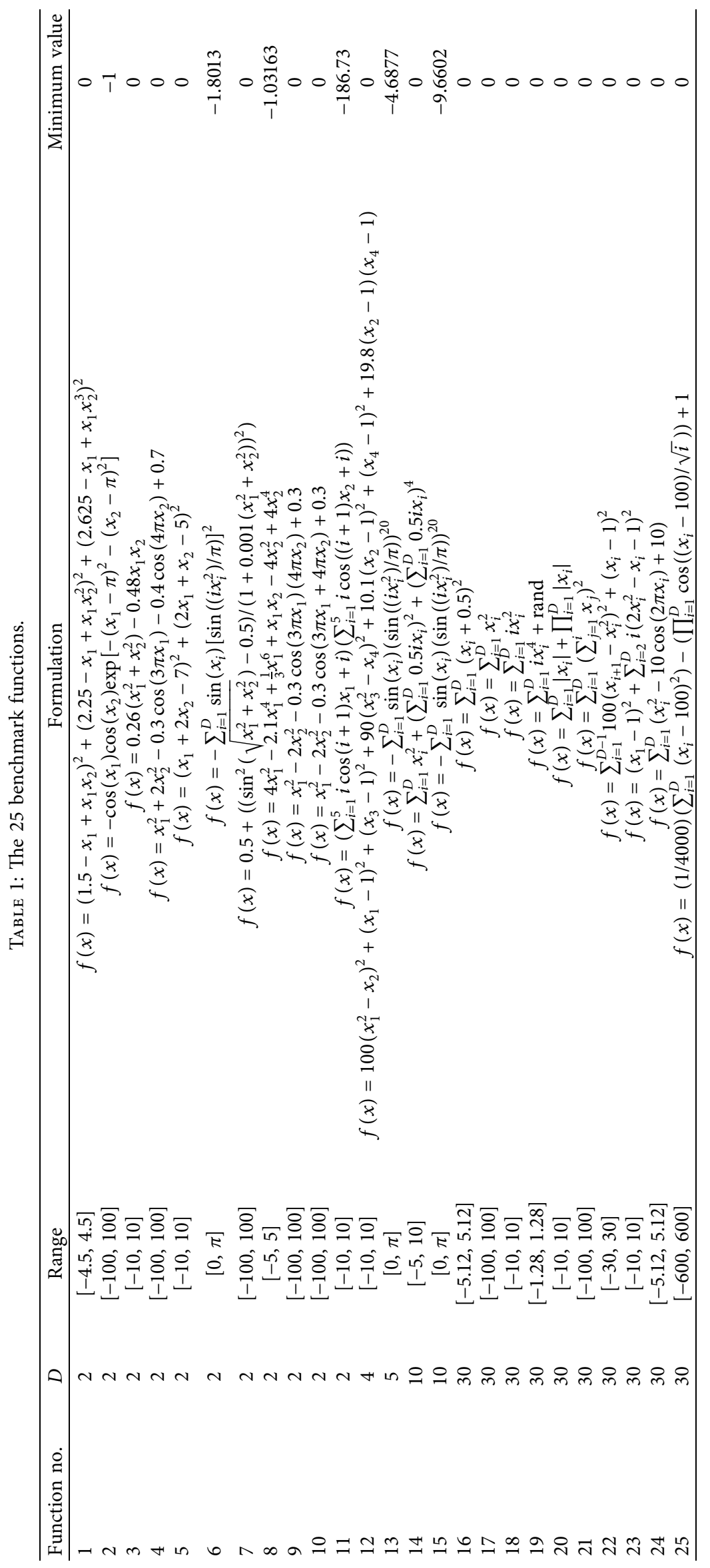


TABLE 2: Compared results of the MSOS with the SOS for the $1^{\text {st }}$ to $15^{\text {th }}$ benchmark functions.

\begin{tabular}{lcccccccccc}
\hline Function & F1 & & F2 & & F3 & \multicolumn{2}{c}{ F4 } & F5 \\
\hline Method & SOS & MSOS & SOS & MSOS & SOS & MSOS & SOS & MSOS & SOS & MSOS \\
Max & 0 & 0 & -1 & -1 & 0 & 0 & 0 & 0 & 0.5072 & 0.5072 \\
Best & 0 & 0 & -1 & -1 & 0 & 0 & 0 & 0 & 0 \\
Mean & 0 & 0 & -1 & -1 & 0 & 0 & 0 & 0 & 0.0101 & 0.0507 \\
STD & 0 & 0 & 0 & 0 & 0 & 0 & 0 & 0 & 0.0717 & 0.1537 \\
Success rate & $100 \%$ & $100 \%$ & $100 \%$ & $100 \%$ & $100 \%$ & $100 \%$ & $100 \%$ & $100 \%$ & $96 \%$ & $90 \%$ \\
Mean con. iter. & 176.7400 & 174.8800 & 74.6600 & 71.6400 & 787.4600 & 786.4200 & 32.0200 & 31.6600 & 261.6600 & 191.4800 \\
Function & F6 & & F7 & & F8 & & F9 & F10 \\
Method & SOS & MSOS & SOS & MSOS & SOS & MSOS & SOS & MSOS & SOS & MSOS \\
Max & -1.8013 & -1.8013 & 0 & 0 & -1.0316 & -1.0316 & 0 & 0 & 0 & 0 \\
Best & -1.8013 & -1.8013 & 0 & 0 & -1.0316 & -1.0316 & 0 & 0 & 0 \\
Mean & -1.8013 & -1.8013 & 0 & 0 & -1.0316 & -1.0316 & 0 & 0 & 0 & 0 \\
STD & 0 & 0 & 0 & 0 & 0 & 0 & 0 & 0 & 0 \\
Success rate & $100 \%$ & $100 \%$ & $100 \%$ & $100 \%$ & $100 \%$ & $100 \%$ & $100 \%$ & $100 \%$ & $100 \%$ & $100 \%$ \\
Mean con. iter. & 78.9000 & 77.2000 & 114.0000 & 115.1000 & 146.9400 & 133.6600 & 30.5000 & 30.4600 & 45.2600 & 45.2600 \\
Function & F11 & & F12 & & F13 & & F14 & F15 \\
Method & SOS & MSOS & SOS & MSOS & SOS & MSOS & SOS & MSOS & SOS & MSOS \\
Max & -186.7298 & -186.7300 & $0.3404 \mathrm{e}-6$ & $0.2422 \mathrm{e}-6$ & -4.6877 & -4.6877 & 0 & 0 & -9.4496 & -9.5606 \\
Best & -186.7300 & -186.7300 & 0 & 0 & -4.6877 & -4.6877 & 0 & 0 & -9.6602 & -9.6602 \\
Mean & -186.7309 & -186.7309 & $0.0213 \mathrm{e}-6$ & $0.0147 \mathrm{e}-6$ & -4.6877 & -4.6877 & 0 & 0 & -9.6192 & -9.6432 \\
STD & 0.0002 & 0.0001 & $0.0530--6$ & $0.0375 \mathrm{e}-6$ & 0 & 0 & 0 & 0 & 0.0573 & 0.0245 \\
Success rate & $12 \%$ & $14 \%$ & $2 \%$ & $2 \%$ & $100 \%$ & $100 \%$ & $100 \%$ & $100 \%$ & $8 \%$ \\
Mean con. iter. & 578.1600 & 555.0000 & 799.1600 & 796.7200 & 312.0000 & 316.5400 & 833.8400 & 833.8200 & 803.7800 & 784.7800 \\
\hline & & & & & & & &
\end{tabular}

TABLE 3: Compared results of the MSOS with the SOS for the $16^{\text {th }}$ to $25^{\text {th }}$ benchmark functions.

\begin{tabular}{|c|c|c|c|c|c|c|c|c|c|c|}
\hline Function & F16 & & F17 & & F18 & & F19 & & $\mathrm{F} 20$ & \\
\hline Method & SOS & MSOS & SOS & MSOS & SOS & MSOS & SOS & MSOS & SOS & MSOS \\
\hline Max & 0 & 0 & 0 & 0 & 0 & 0 & $0.6300 \mathrm{e}-03$ & $0.8483 \mathrm{e}-03$ & 0 & 0 \\
\hline Best & 0 & 0 & 0 & 0 & 0 & 0 & $0.0947 \mathrm{e}-03$ & $0.0873 e-03$ & 0 & 0 \\
\hline Mean & 0 & 0 & 0 & 0 & 0 & 0 & $0.3399 \mathrm{e}-03$ & $0.2882 \mathrm{e}-03$ & 0 & 0 \\
\hline STD & 0 & 0 & 0 & 0 & 0 & 0 & $0.1313 \mathrm{e}-03$ & $0.1376 \mathrm{e}-03$ & 0 & 0 \\
\hline Success rate & $100 \%$ & $100 \%$ & $100 \%$ & $100 \%$ & $100 \%$ & $100 \%$ & $2 \%$ & $2 \%$ & $100 \%$ & $100 \%$ \\
\hline Mean con. iter. & 714.6400 & 712.0200 & 833.9400 & 833.9600 & 833.9600 & 833.9400 & 612.7600 & 591.5800 & 833.9600 & 833.8600 \\
\hline Function & $\mathrm{F} 21$ & & $\mathrm{~F} 22$ & & $\mathrm{~F} 23$ & & $\mathrm{~F} 24$ & & $\mathrm{~F} 25$ & \\
\hline Method & SOS & MSOS & SOS & MSOS & SOS & MSOS & SOS & MSOS & SOS & MSOS \\
\hline $\operatorname{Max}$ & 0 & 0 & 21.5087 & 22.5117 & 0.6667 & 0.6667 & 0 & 0 & 0 & 0 \\
\hline Best & 0 & 0 & 15.0510 & 15.4677 & 0.6667 & 0.6667 & 0 & 0 & 0 & 0 \\
\hline Mean & 0 & 0 & 18.1114 & 18.2658 & 0.6667 & 0.6667 & 0 & 0 & 0 & 0 \\
\hline STD & 0 & 0 & 1.4150 & 1.5185 & 0 & 0 & 0 & 0 & 0 & 0 \\
\hline Success rate & $100 \%$ & $100 \%$ & $2 \%$ & $2 \%$ & $100 \%$ & $100 \%$ & $100 \%$ & $100 \%$ & $100 \%$ & $100 \%$ \\
\hline Mean con. iter. & 833.9800 & 833.9800 & 833.8400 & 833.6400 & 435.5200 & 453.6600 & 89.4400 & 94.2000 & 74.1600 & 74.1000 \\
\hline
\end{tabular}

TABLE 4: Control parameters of the MSOS and the SOS for the distribution systems.

\begin{tabular}{|c|c|c|c|c|}
\hline Item & 14-nodes & 33-nodes & 69-nodes & 119-nodes \\
\hline Population size & 5 & 10 & 10 & 20 \\
\hline Problem dimension & 3 & 5 & 5 & 15 \\
\hline Maximum number of evaluations & 500 & 2000 & 2000 & 20000 \\
\hline
\end{tabular}




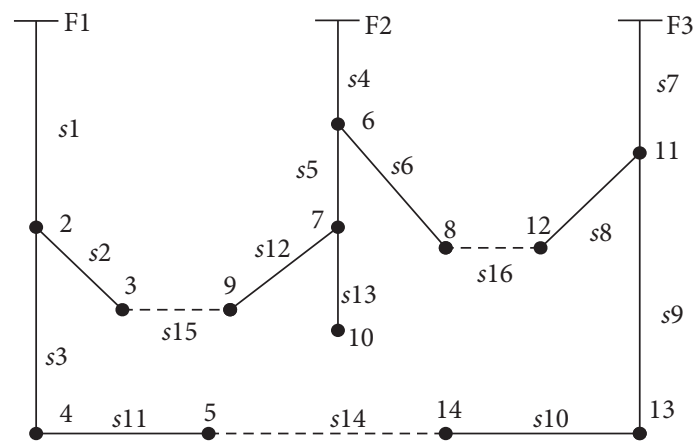

Figure 3: The 14-node test system.

TABle 5: The optimal configuration obtained by the MSOS for the 14-node system.

\begin{tabular}{lcccc}
\hline Method & Open SW & Power loss (kW) & Loss reduction (\%) & Minimum voltage (p.u.) \\
\hline Initial & $14,15,16$ & 511.4356 & 8.86 & 0.9693 \\
MSOS & $6,12,14$ & 466.1267 & 8.86 & 0.9716 \\
SOS & $6,12,14$ & 466.1267 & 8.86 & 0.9716 \\
MTS [39] & $6,12,14$ & 466.1267 & 8.86 & 0.9716 \\
GA [5] & $6,12,14$ & 466.1267 & 8.86 & 0.9716 \\
\hline
\end{tabular}

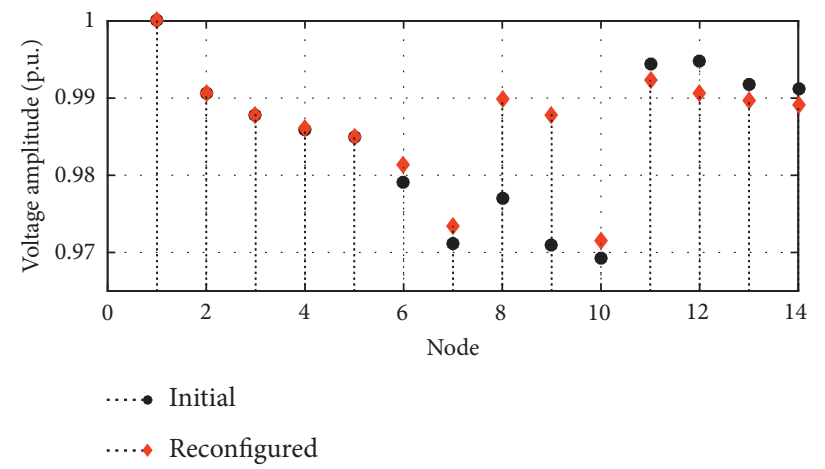

Figure 4: Voltage amplitude of the nodes for the 14-node test system.

TABLE 6: Comparison results between the MSOS and the SOS for the 14-node system over 50 independent runs.

\begin{tabular}{lcc}
\hline Item & MSOS & SOS \\
\hline Optimal solution & $6,12,14$ & $6,12,14$ \\
Power loss (kW) & 466.1267 & 466.1267 \\
Minimum voltage (p.u.) & 0.9716 & 0.9716 \\
Maximum fitness value & 466.1267 & 493.1542 \\
Minimum fitness value & 466.1267 & 466.1267 \\
Average fitness value & 466.1267 & 467.1939 \\
STD of fitness & 0 & 4.5628 \\
Success rate (\%) & 100 & 94 \\
Number of average convergence iterations & 6.14 & 5.82 \\
STD of the number of average convergence iterations & 4.7294 & 4.0390 \\
Running time (second) & 1.5134 & 1.5016 \\
\hline
\end{tabular}


by applying the proposed MSOS technique. This minimum power loss value is obtained by opening the new switches of $\{\mathrm{s} 6, \mathrm{~s} 12$, and $\mathrm{s} 14\}$. The voltage profile of the system achieved by the proposed MSOS technique is shown in Figure 4. From the figure, it can be seen that most of the nodes have a significant voltage improvement. The minimum voltage amplitude has been increased from 0.9693 p.u. (before reconfiguration) to $0.9716 \mathrm{p} . \mathrm{u}$. (after reconfiguration) by the MSOS. As also shown in Table 5, the results achieved by the MSOS are identical to those of the SOS, modified tabu search (MTS) [39], and GA [5].

The comparison results between the MSOS and the SOS for the 14-node system over 50 independent runs are shown in Table 6. From the table, the MSOS has identified the optimal solution for all of runs, whereas the success rate is only $94 \%$ for the SOS. The average value of the fitness function in 50 runs of the MSOS is identical to the minimum value of the fitness function. The STD of the fitness function is equal to zero; it means that the fitness function value in each run is equal to the average value of the fitness function in 50 runs. Meanwhile, for the SOS, the maximum, average, and STD values of the fitness function are 493.1542, 467.1939 , and 4.5628, respectively. All of these results are higher than those of the MSOS. The average running time for each run of both the methods is nearly equal, and it is about 1.5134 seconds for the MSOS and 1.5016 seconds for the SOS. The average convergence trends of the proposed MSOS and SOS in 50 runs are shown in Figure 5. As shown in the figure, the average convergence trend of the MSOS converges to the best fitness value ( fit $\left._{\text {best }}\right)$, whereas the SOS converges to the higher value compared with the MSOS. These results have shown that the network reconfiguration based on the MSOS for the 14-node test system outperforms the original SOS.

5.2.2. The 33-Node Test System. The $12.66 \mathrm{kV}, 33$-node test system shown in Figure 6 contains five initial open switches of $\{s 33, s 34, s 35, s 36$, and s37\}. The power loss and the minimum voltage amplitude are $202.6863 \mathrm{~kW}$ and 0.9131 p.u., respectively. The parameters of the branches and the nodes are referenced in [41].

The network reconfiguration results of the 33-node system are shown in Table 7. After reconfiguration, the power loss has been reduced from 202.6863 to $139.9823 \mathrm{~kW}$, corresponding to $30.94 \%$ of the power loss reduction. The minimum voltage amplitude has also been raised from 0.9131 to 0.94124 p.u. after reconfiguration by the MSOS. The voltage profile of the system after reconfiguration compared to the initial configuration in Figure 7 shows that most of the voltage amplitude of nodes has been enhanced after reconfiguration. The positive results of power loss reduction and voltage profile improvement are gained by opening the switches of $\{s 7, s 9, s 14, s 28$ and s32\}. The optimal solution obtained by using the MSOS is identical to that of SOS, ICSA [14], CSA [15], and FWA [20], and better than that of the improved tabu search (ITS) [42] and the harmony search algorithm (HSA) [42]. Power loss caused by the optimal solutions of ITS and HSA is, respectively, 2.1877 and $2.4477 \mathrm{~kW}$ higher than that of the MSOS. In addition, the minimum voltage amplitude of the above methods is also lower than that of the MSOS. Compared with RRA [18], GA [42], IAICA [19], RGA [5], and WGEMA [21], power loss obtained by the MSOS is $0.4323 \mathrm{~kW}$ higher, but the minimum voltage amplitude is 0.0034 p.u. higher than that of the aforementioned methods.

The comparison results between the MSOS and the SOS for the 33-node system over 50 independent runs are shown in Table 8. From the table, the MSOS has identified the optimal solution with a success rate of $88 \%$, whereas it is only $76 \%$ for the SOS. The average fitness value of the MSOS is equal to 149.2748 , which is 1.0229 lower than that of the SOS. In addition, the STD of the fitness function value in 50 trials is also much lower than that of the SOS. The average running time for each run of the two methods is approximately equal, and it is about 4.6144 seconds for the MSOS and 4.3819 seconds for the SOS. The average convergence trends of the proposed MSOS and SOS in 50 runs for the 33node system are shown in Figure 8. As shown in the figure, in 50 runs, the average convergence trend of the MSOS converges to the best fitness value, whereas the SOS converges to the higher value compared to the MSOS. These results have shown that the MSOS gains more positive results than the SOS.

5.2.3. The 69-Node Test System. The 69-node test system demonstrated in Figure 9 comprises five open switches and 68 load nodes [43]. The power loss of the system is $224.8871 \mathrm{~kW}$, which is caused by opening the switches of $\{s 69, s 70, s 71, s 72$, and $s 73\}$. After reconfiguration, the switches consisting of $\{s 14, s 57, s 61, s 69$, and $s 70\}$ are opened to substitute for the initial open switches. At that time, the power loss of the system is only $98.5875 \mathrm{~kW}$, which is $126.2966 \mathrm{~kW}$ lower than that of the initial configuration. Furthermore, the minimum voltage amplitude after reconfiguration is 0.9495 p.u., which is much higher than 0.9092 p.u. of the initial configuration. The voltage amplitudes of all nodes shown in Figure 10 have been significantly improved after performing reconfiguration by the MSOS. As shown in Table 9, power loss obtained by the MSOS is identical to that of SOS, ICSA [14], CSA [15], ASFLA [16], FWA [44], IAICA [19], backtraking seach algorithm (BSA) [46], and WGEMA [21], and better than the result obtained by HSA [45].

The comparison results between the MSOS and the SOS for the 69-node system are shown in Table 10. From the table, the MSOS has identified the optimal solution with a higher success rate, compared to the SOS. In more detail, in 50 trials, the MSOS has reached the optimal solution 35 times corresponding to $70 \%$ of the runs, whereas this number is only $46 \%$ for the original SOS. In addition, the 


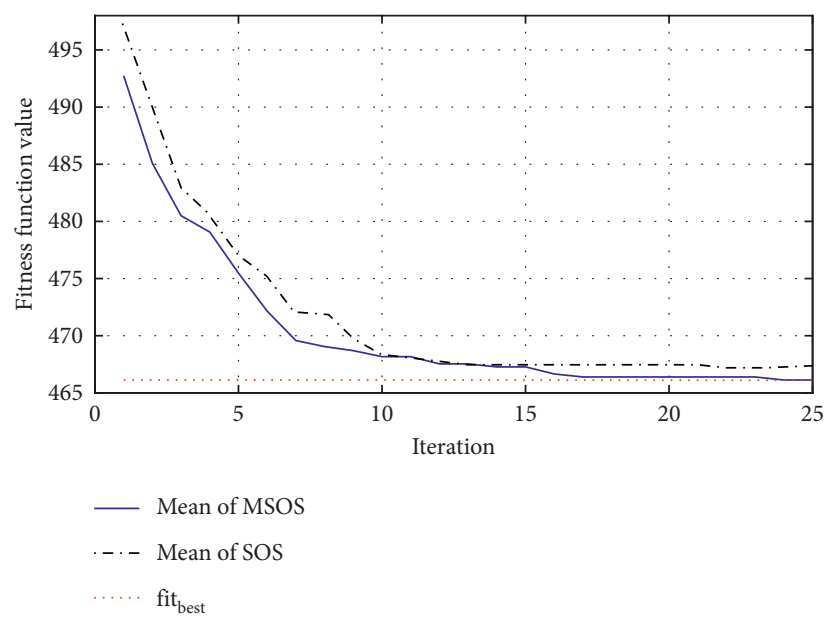

Figure 5: Mean convergence characters of the MSOS and the SOS for the 14-node test system.

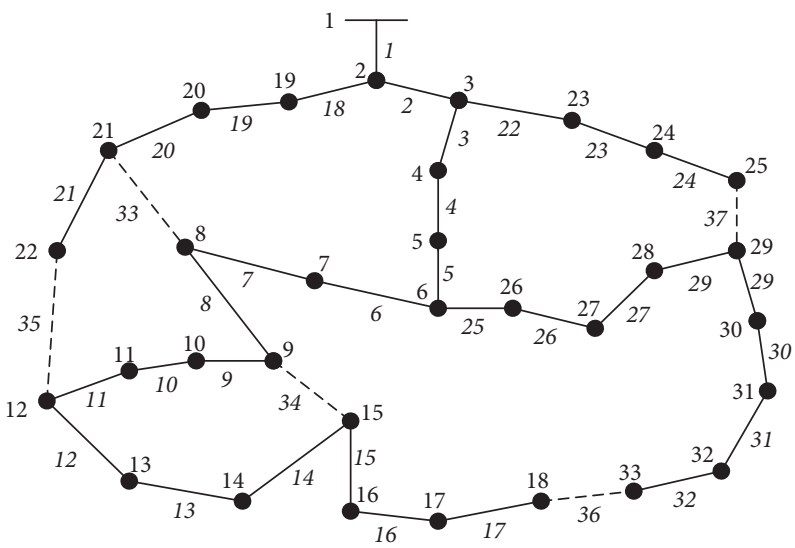

FIgURE 6: The 33-node test system.

TABLE 7: The optimal configuration obtained by MSOS for the 33-node system.

\begin{tabular}{lcccc}
\hline Method & Open SW & Power loss $(\mathrm{kW})$ & Loss reduction (\%) & Minimum voltage (p.u.) \\
\hline Initial & $33,34,35,36,37$ & 202.6863 & - & 0.9131 \\
MSOS & $7,9,14,28,32$ & 139.9823 & 30.94 & 0.9412 \\
SOS & $7,9,14,28,32$ & 139.9823 & 30.94 & 0.9412 \\
ICSA [14] & $7,9,14,28,32$ & 139.9823 & 30.94 & 0.9412 \\
CSA [15] & $7,9,14,28,32$ & 139.9823 & 31.15 & 0.9412 \\
RRA [18] & $7,14,9,32,37$ & 139.55 & 30.94 & 0.9378 \\
FWA [20] & $7,9,14,28,32$ & 139.9823 & 31.15 & 0.9412 \\
GA [42] & $7,9,14,32,37$ & 139.55 & 29.86 & 0.9378 \\
ITS [42] & $7,9,14,36,37$ & 142.17 & 31.15 & 0.9336 \\
IAICA [19] & $7,9,14,32,37$ & 139.55 & 29.73 & 0.9378 \\
HSA [42] & $7,10,14,28,36$ & 142.43 & 31.15 & 0.9377 \\
RGA [5] & $7,9,14,32,37$ & 31.15 & 0.9378 \\
WGEMA [21] & $7,9,14,32,37$ & 139.55 & 0.9378 \\
\hline
\end{tabular}

average and STD values of the fitness function in 50 runs of the MSOS are 99.7868 and 1.8434 , respectively, which are lower by 0.97323 and 0.6576 , respectively, compared to those of the SOS. The number of average convergence iterations of both the methods is nearly the same. The calculated time of the MSOS is slightly higher compared to the SOS, but the 


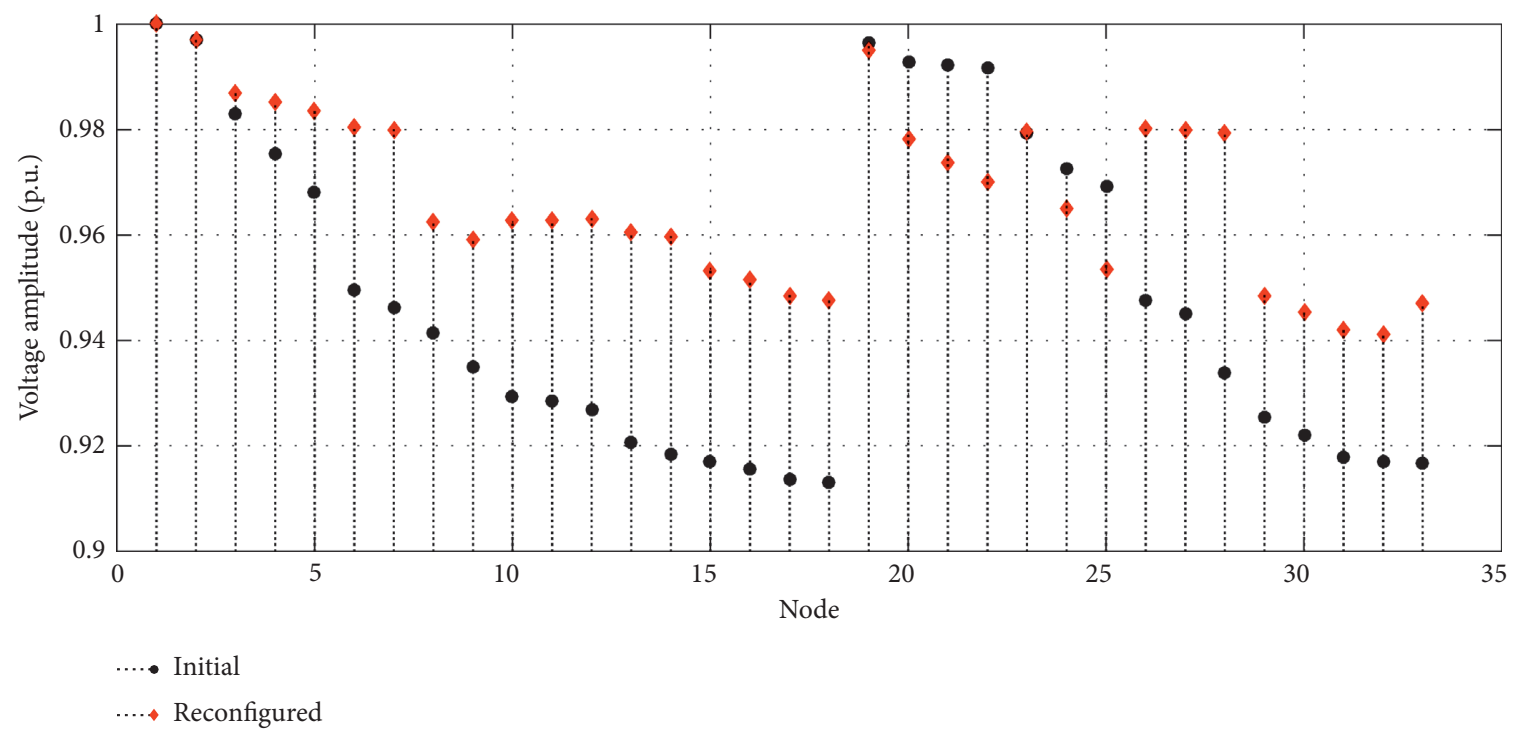

Figure 7: Voltage amplitude of the nodes for the 33-nodes test system.

TABLE 8: Comparison results between the MSOS and the SOS for the 33-node system over 50 independent runs.

\begin{tabular}{lcc}
\hline Item & MSOS & SOS \\
\hline Optimal solution & $7,9,14,28,32$ & $7,9,14,28,32$ \\
Power loss (kW) & 139.9823 & 139.9823 \\
Minimum voltage (p.u.) & 0.94124 & 0.94124 \\
Maximum fitness value & 156.4541 & 159.5083 \\
Minimum fitness value & 148.7392 & 148.7392 \\
Average fitness value & 149.2748 & 150.2977 \\
STD of fitness & 1.6522 & 3.0475 \\
Success rate (\%) & 88 & 76 \\
Number of average convergence iterations & 27.18 & 22.96 \\
STD of number of average convergence iterations & 11.6052 & 9.9097 \\
Running time (second) & 4.6144 & 4.3819 \\
\hline
\end{tabular}

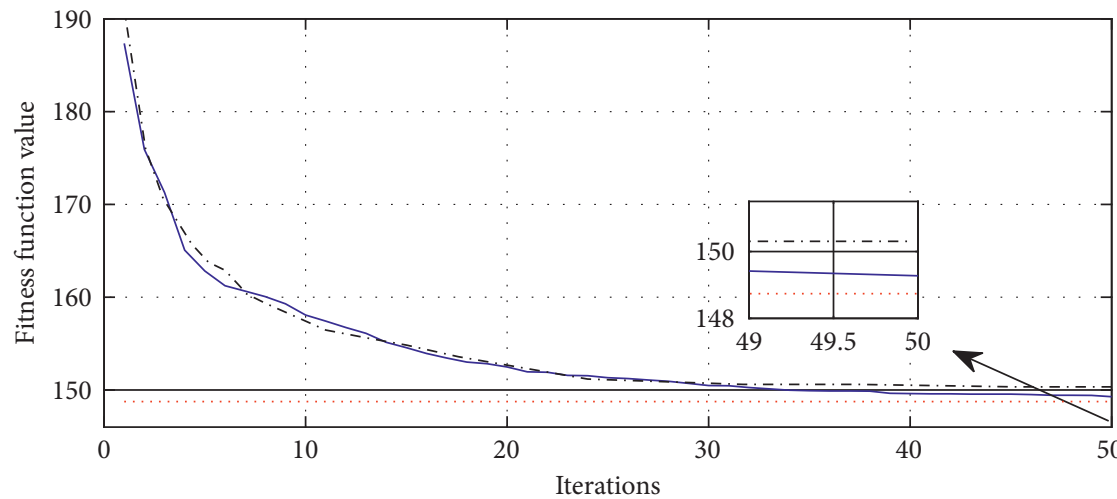

— Mean of MSOS

-.- Mean of SOS

fit $_{\text {best }}$

Figure 8: Mean convergence characters of the MSOS and the SOS for the 33-node test system. 


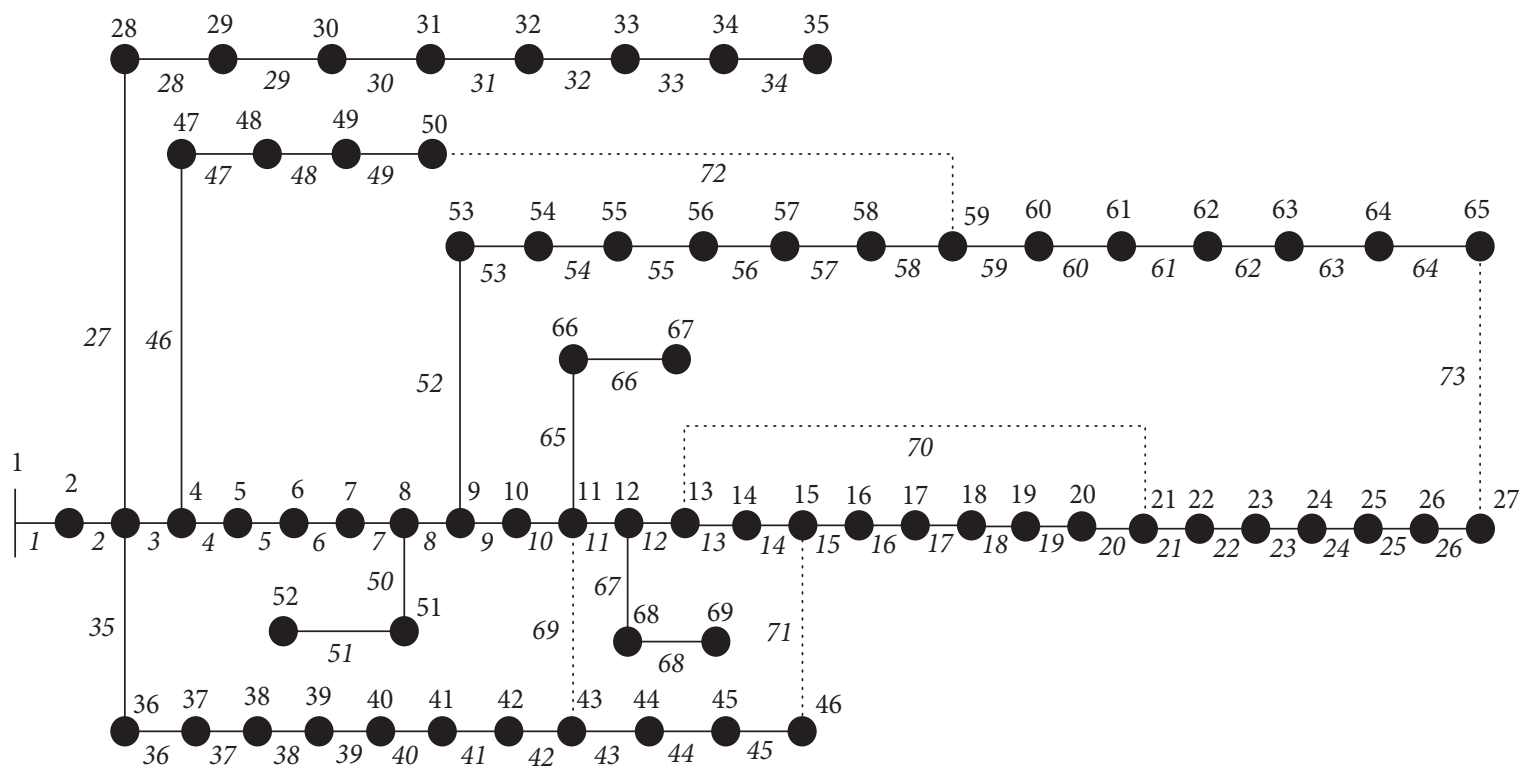

FIgURE 9: The 69-node test system.

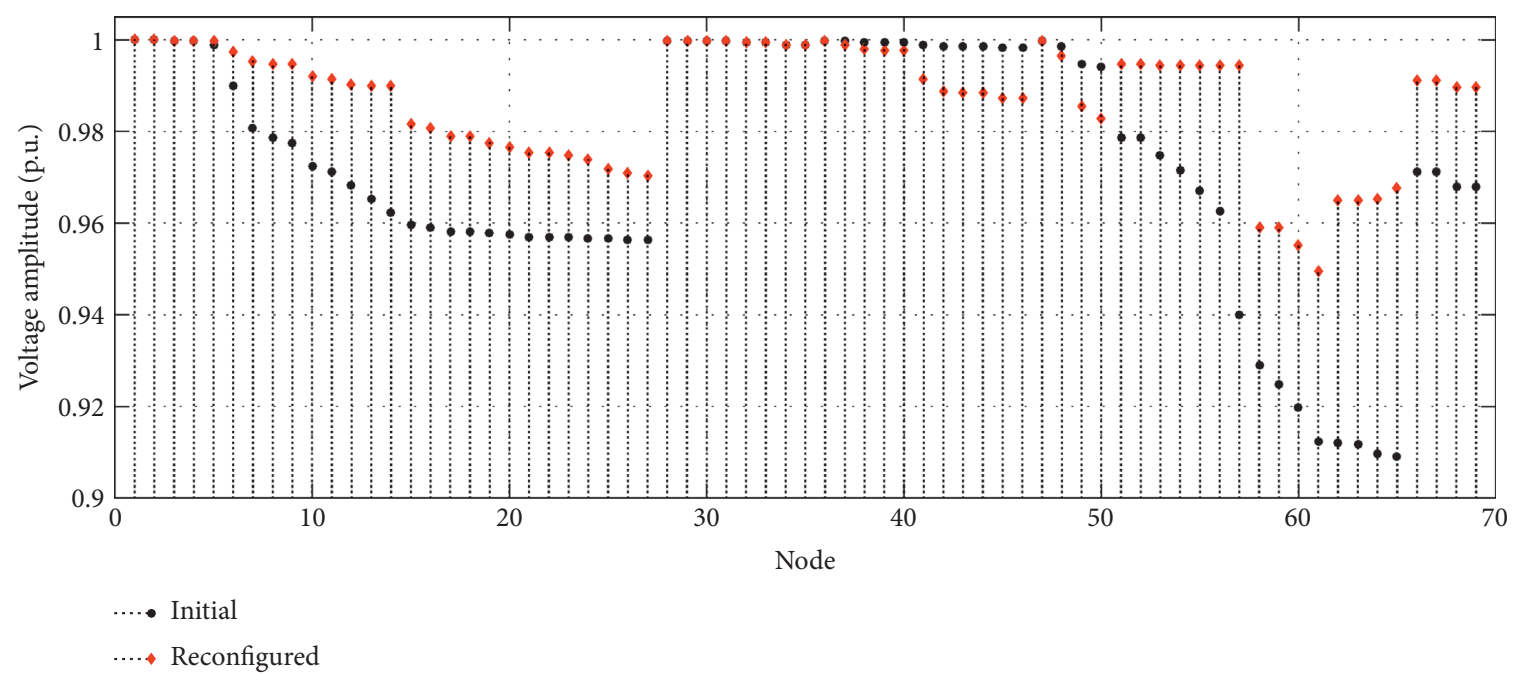

Figure 10: Voltage amplitude of the nodes for the 69-node test system.

TABLE 9: The optimal configuration obtained by the MSOS for the 69-node system.

\begin{tabular}{lcccc}
\hline Method & Open SW & Power loss $(\mathrm{kW})$ & Loss reduction (\%) & Minimum voltage (p.u.) \\
\hline Initial & $69,70,71,72,73$ & 224.8871 & - & 0.9092 \\
MSOS & $14,57,61,69,70$ & 98.5875 & 56.16 & 0.9495 \\
SOS & $14,57,61,69,70$ & 98.5875 & 56.16 & 0.9495 \\
ICSA [14] & $69,70,14,57,61$ & 98.59 & 56.16 & 0.9495 \\
CSA [15] & $14,57,61,69,70$ & 98.59 & 56.16 & 0.9495 \\
ASFLA [16] & $14,56,61,69,70$ & 98.59 & 56.16 & 0.9495 \\
FWA [44] & $14,56,61,69,70$ & 98.59 & 53.23 & 0.9495 \\
HSA [45] & $13,18,56,61,69$ & 105.19 & 56.16 & 0.9495 \\
IAICA [19] & $69,70,14,57,61$ & 98.59 & 56.16 & 0.9495 \\
BSA [46] & $14,57,61,69,70$ & 98.5875 & 56.16 & 0.9495 \\
WGEMA [21] & $14,56,61,69,70$ & 98.59 & 0.9495 \\
\hline
\end{tabular}


TABLe 10: Comparison results between the MSOS and the SOS for the 69-node system over 50 independent runs.

\begin{tabular}{lcc}
\hline Item & MSOS & SOS \\
\hline Optimal solution & $14,57,61,69,70$ & $14,57,61,69,70$ \\
Power loss (kW) & 98.5875 & 98.5875 \\
Minimum voltage (p.u.) & 0.9495 & 0.9495 \\
Maximum fitness value & 106.5732 & 105.6075 \\
Minimum fitness value & 99.1169 & 99.1169 \\
Average fitness value & 99.7868 & 160.7601 \\
STD of fitness & 1.8434 & 2.5010 \\
Success rate (\%) & 70 & 46 \\
Number of average convergence iterations & 33.62 & 34.46 \\
STD of number of average convergence iterations & 10.548 & 10.1824 \\
Running time (second) & 15.3756 & 13.3928 \\
\hline
\end{tabular}

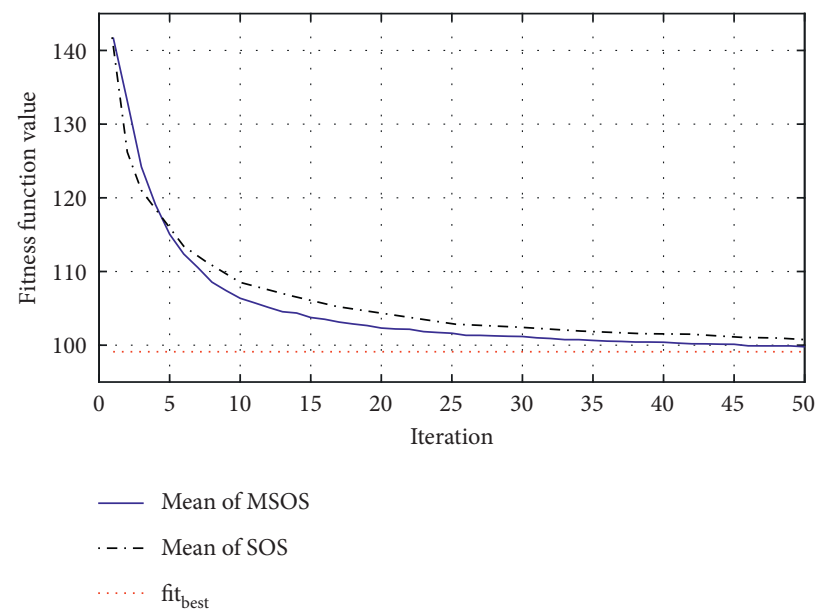

FIGURE 11: Mean convergence characters of the MSOS and the SOS for the 69-nodes test system.

total time of 15.3756 seconds is suitable for a medium-scale system such as the 69-node test system. The average convergence trends of the MSOS and the SOS in 50 runs are shown in Figure 11. As shown in the figure, in 50 runs, the average convergence trend of the MSOS converges to the lower value compared to the SOS. These results have again shown that the MSOS outperforms the SOS in the NR problem.

5.2.4. The 119-Node Test System. The initial configuration of the system comprises 15 open switches of $\{118,119,120,121$, $122,123,124,125,126,127,128,129,130,131,132\}$, as shown in Figure 12. These open switches cause a total loss of $1273.45 \mathrm{~kW}$ and a minimum voltage amplitude per unit of 0.8678 [47]. As shown in Table 11, the optimal configuration obtained by the MSOS causes a power loss of $855.0402 \mathrm{~kW}$ corresponding to $32.86 \%$ loss reduction. The minimum voltage amplitude per unit is 0.9298 , which is 0.062 higher than that of the initial configuration. Furthermore, nodes' amplitude has been significantly enhanced after reconfiguration, as demonstrated in Figure 13. Table 11 has also demonstrated that the optimal solution achieved by the MSOS is identical to SOS, ICSA [14], CSA [15], and FWA [44], but better than that of MTS [39] and ITS [47]. In more detail, the total loss obtained by the MTS and the ITS is $12.3599 \mathrm{~kW}$ higher than that of the proposed MSOS method.

The comparison results between the MSOS and the SOS for the 119-node system over 50 independent runs are shown in Table 12. From the table, although the MSOS and SOS methods have found the optimal solution with low success rates, the effectiveness of the MSOS is still more significant than the SOS. The MSOS has identified the optimal solution with a success rate of $24 \%$, whereas it is only $14 \%$ for the SOS. The average fitness value of the MSOS is lower than that of the SOS. In addition, the STD of the fitness function value in 50 trials is also much lower than that of the SOS. The average running time for each run of the MSOS is about 10 seconds higher than that of the SOS. The average convergence trends of the proposed MSOS and SOS in 50 runs for the 119-node system are shown in Figure 14. As shown in the figure, in 50 runs, the average convergence trend of the MSOS converges to the best fitness value, whereas the SOS converges to a higher value compared with 


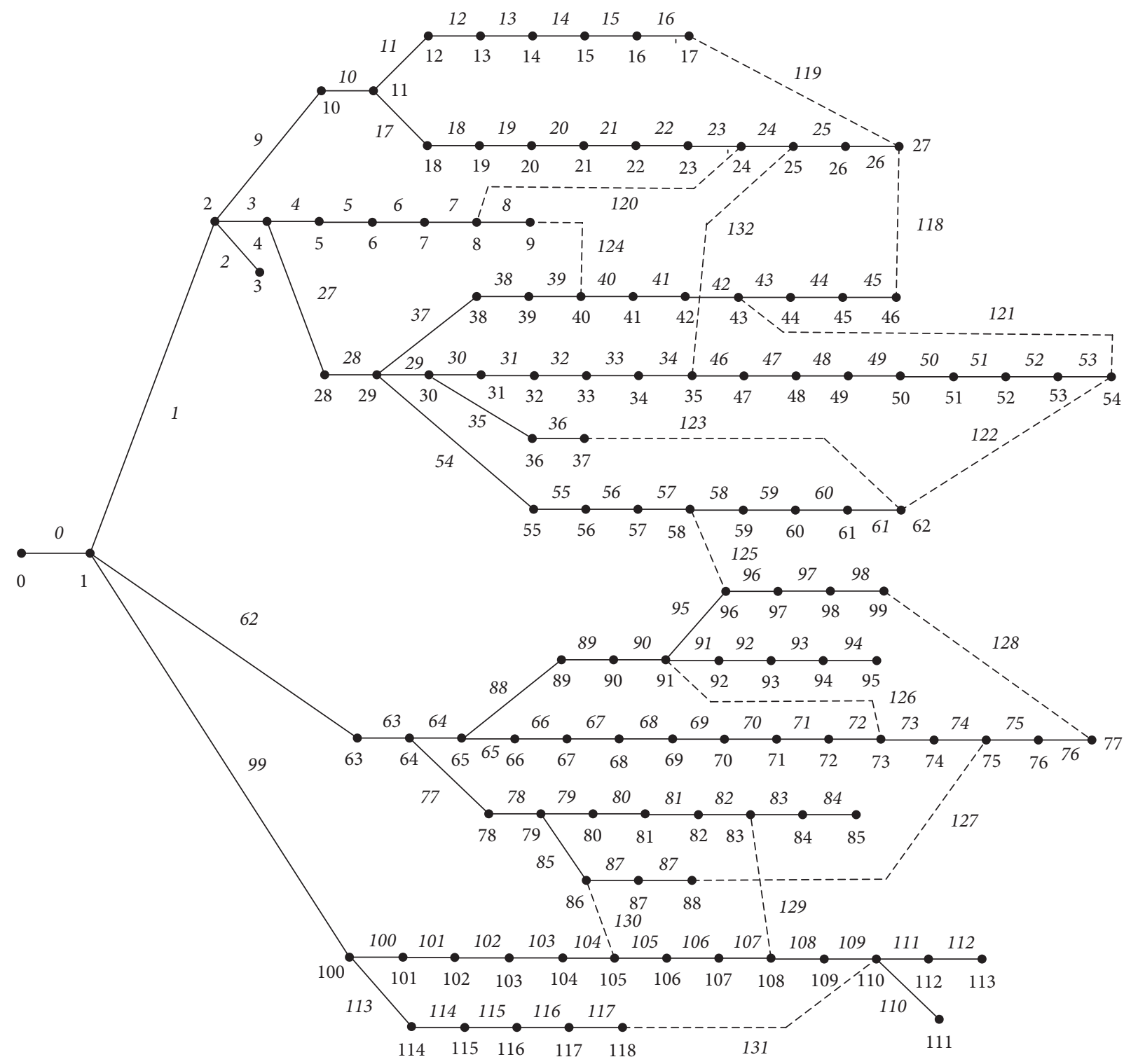

Figure 12: The 119-node test system.

TABle 11: The optimal configuration obtained by the MSOS for the 119-node system.

\begin{tabular}{|c|c|c|c|c|}
\hline Method & Open SW & $\begin{array}{l}\text { Power loss } \\
(\mathrm{kW})\end{array}$ & $\begin{array}{l}\text { Loss reduction } \\
(\%)\end{array}$ & Minimum voltage (p.u.) \\
\hline Initial & $\begin{array}{c}118,119,120,121,122,123,124,125,126,127,128,129,130 \\
131,132\end{array}$ & 1273.45 & - & 0.8678 \\
\hline MSOS & $23,25,34,39,42,50,58,71,74,95,97,109,121,129,130$ & 855.0402 & 32.86 & 0.9298 \\
\hline SOS & $23,25,34,39,42,50,58,71,74,95,97,109,121,129,130$ & 855.0402 & 32.86 & 0.9298 \\
\hline ICSA [14] & $23,25,34,39,42,50,58,71,74,95,97,109,121,129,130$ & 855.04 & 32.86 & 0.9298 \\
\hline CSA [15] & $42,25,23,121,50,58,39,95,71,74,97,129,130,109,34$ & 855.04 & 32.86 & 0.9298 \\
\hline $\begin{array}{l}\text { FWA } \\
{[44]}\end{array}$ & $23,25,34,39,42,50,58,71,74,95,97,109,121,129,130$ & 855.04 & 32.86 & 0.9298 \\
\hline ITS [47] & $42,26,23,51,122,58,39,95,71,74,97,129,130,109,34$ & 867.4 & 31.89 & 0.9298 \\
\hline MTS [39] & $42,26,23,51,122,58,39,95,71,74,97,129,130,109,34$ & 867.4 & 31.89 & 0.9298 \\
\hline
\end{tabular}




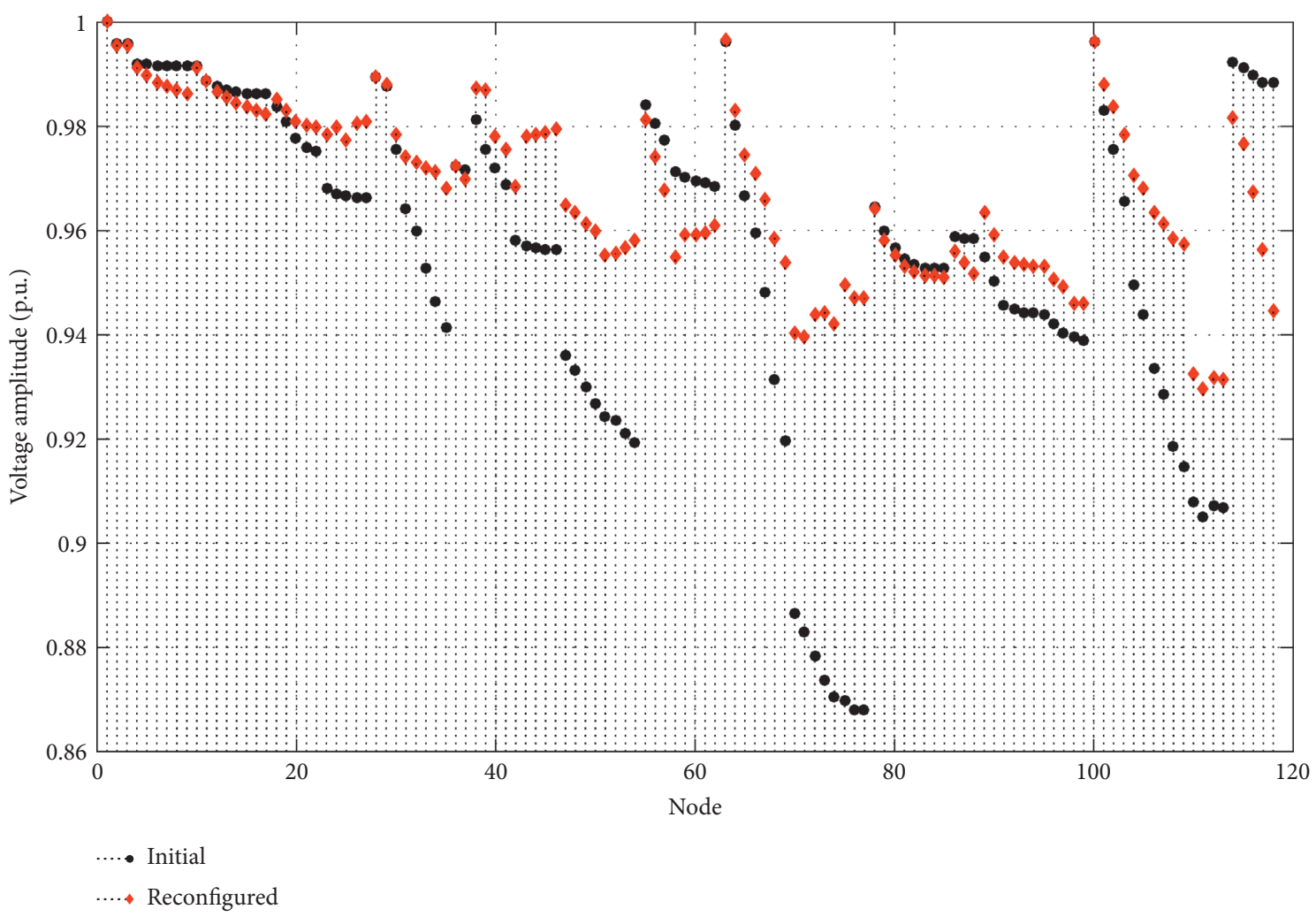

FIGURE 13: Voltage amplitude of nodes for the 119-node test system.

TABLE 12: Comparison of results between the MSOS and the SOS for the 119-node system over 50 independent runs.

\begin{tabular}{lcc}
\hline Item & MSOS & SOS \\
\hline Optimal solution & $23,25,34,39,42,50,58,71,74,95,97,109,23,25,34,39,42,50,58,71,74,95,97,109$, \\
$121,129,130$ \\
Power loss (kW) & $121,129,130$ & 855.0402 \\
Minimum voltage (p.u.) & 855.0402 & 0.92975 \\
Maximum fitness value & 0.92975 & 967.3838 \\
Minimum fitness value & 915.7986 & 875.2876 \\
Average fitness value & 875.2876 & 898.0087 \\
STD of fitness & 891.3358 & 17.904 \\
Success rate (\%) & 11.4839 & 14 \\
Number of average convergence iterations & 24 & 212.68 \\
STD of the number of average convergence & 141.38 & 30.6297 \\
iterations & 43.3372 & 130.2631 \\
Running time (second) & 158.1175 & \\
\hline
\end{tabular}

the MSOS. These results have shown that the MSOS gains more positive results than the SOS for the large-scale test system.

5.2.5. The Impact of Two Modifications of the MSOS for the NR Problem. In order to evaluate the effect of the modification techniques of the MSOS for the NR problem, each modification technique, in turn, is equipped for SOS including SOS with the first modification technique (called SOS-1), an SOS with the second modification technique (known as SOS-2) and MSOS using both of the modification techniques. All four methods including SOS, SOS-1, SOS-2, and MSOS are used to find the optimal configuration for the 119-node test system. The ability of each algorithm is compared with each other through indicators such as maximum, minimum and average fitness values; STD of 


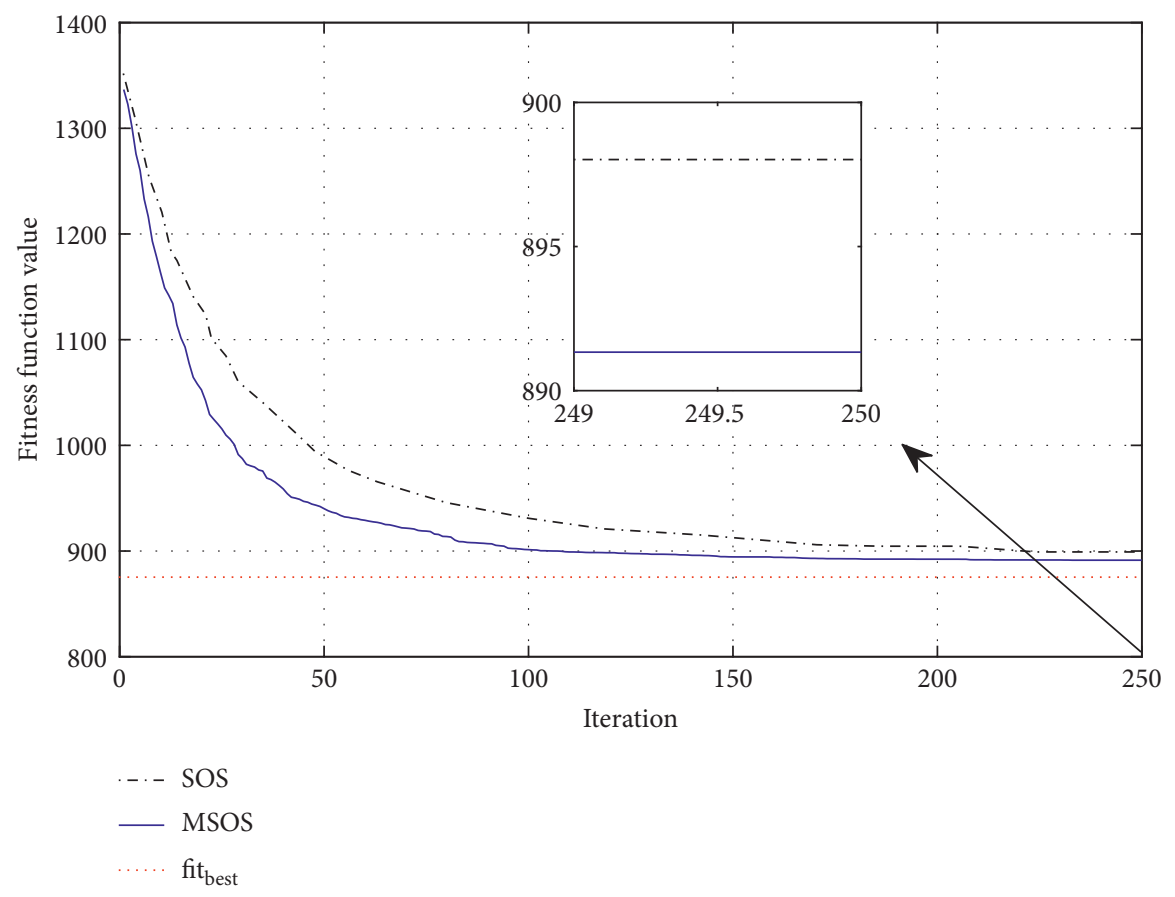

Figure 14: Mean convergence characters of the MSOS and the SOS for the 119-nodes test system.

TABLE 13: Impact of the two modifications of the MSOS to the NR results for the 119-node system.

\begin{tabular}{|c|c|c|c|c|}
\hline Item & SOS & SOS-1 & SOS-2 & MSOS \\
\hline Maximum fitness value & 967.3838 & 930.7066 & 931.1447 & 915.7986 \\
\hline Minimum fitness value & 875.2876 & 875.2876 & 875.2876 & 875.2876 \\
\hline Average fitness value & 898.0087 & 892.71 & 891.454 & 891.3358 \\
\hline STD of fitness & 17.904 & 12.0549 & 13.7885 & 11.4839 \\
\hline Success rate $(\%)$ & 14 & 16 & 20 & 24 \\
\hline Number of average convergence iterations & 212.68 & 166.64 & 144.66 & 141.38 \\
\hline STD of the number of average convergence iterations & 30.6297 & 53.1201 & 42.2879 & 43.3372 \\
\hline Running time (second) & 130.2631 & 140.9075 & 157.6863 & 158.1175 \\
\hline Mean of first-count & 0 & 21.0200 & 0 & 21.4000 \\
\hline Mean of second-count & 77.3200 & 64.4000 & 94.7400 & 91.7800 \\
\hline
\end{tabular}

fitness function value; success rate; number of average convergence iterations; and running time. In addition, to validate the effects of the first modification technique, a count variable (called the first-count) is used to count the number of times that the best so-far organism is updated after using the mutualism strategy. Meanwhile, in order to evaluate the effects of the second modification technique, another counter variable (called the second-count) is used to count the number of times that the new organism generated by the parasitism strategy is better than the jth current organism. In other words, this variable is used to count the number of times that the new organism has the better quality than the current one.
The results obtained by the four methods are shown in Table 13. For SOS-1, by using the first modification technique, the best so-far organism has improved by about 21.0200 times for each run. Similarly, for the MSOS, the first modification technique has helped the best so-far organism that is updated up to about 21.4000 times as soon as the mutualism strategy is implemented. The value of the firstcount value in each run of the SOS- 1 and the MSOS is shown in Figure 15. The figure shows that in each run, the best organism can be updated after using the mutualism strategy. Thus, using the first modification technique will ensure that the best so-far organism used in the commensalism strategy is the best one in the current population. 


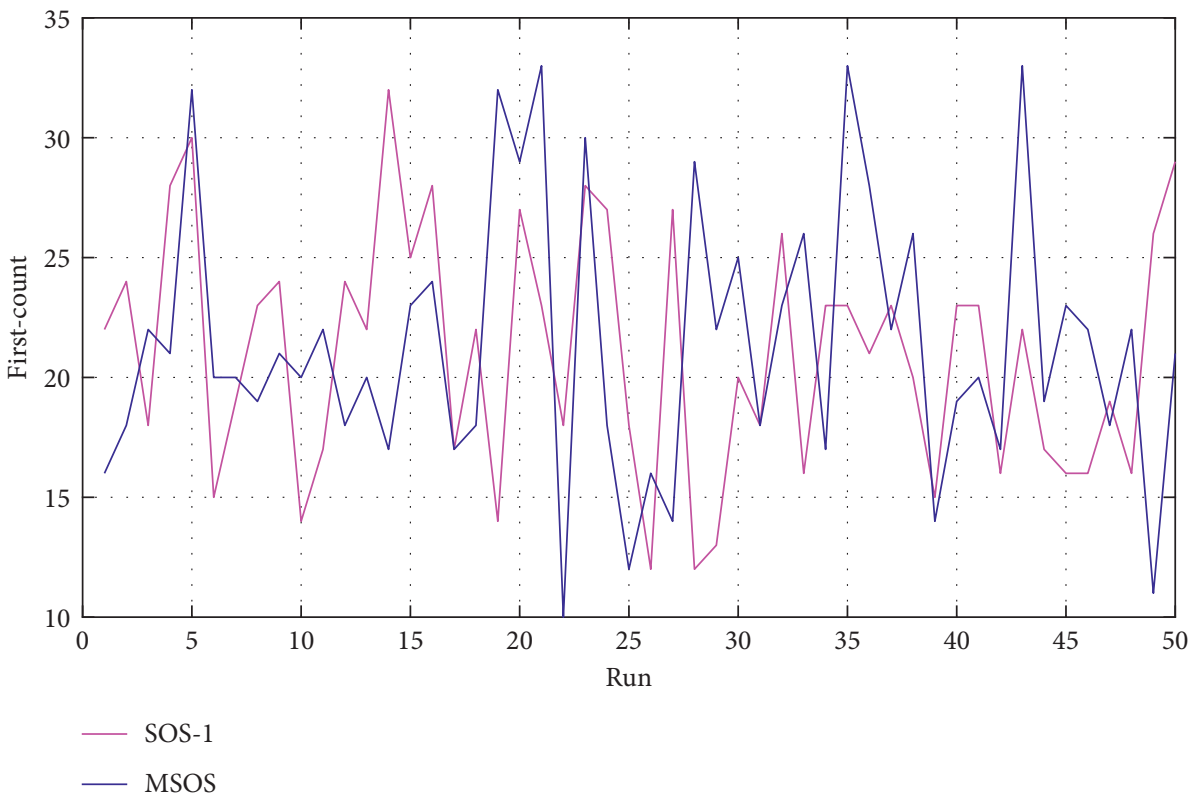

Figure 15: Number of times the best so-far organism is updated by the first modification technique.

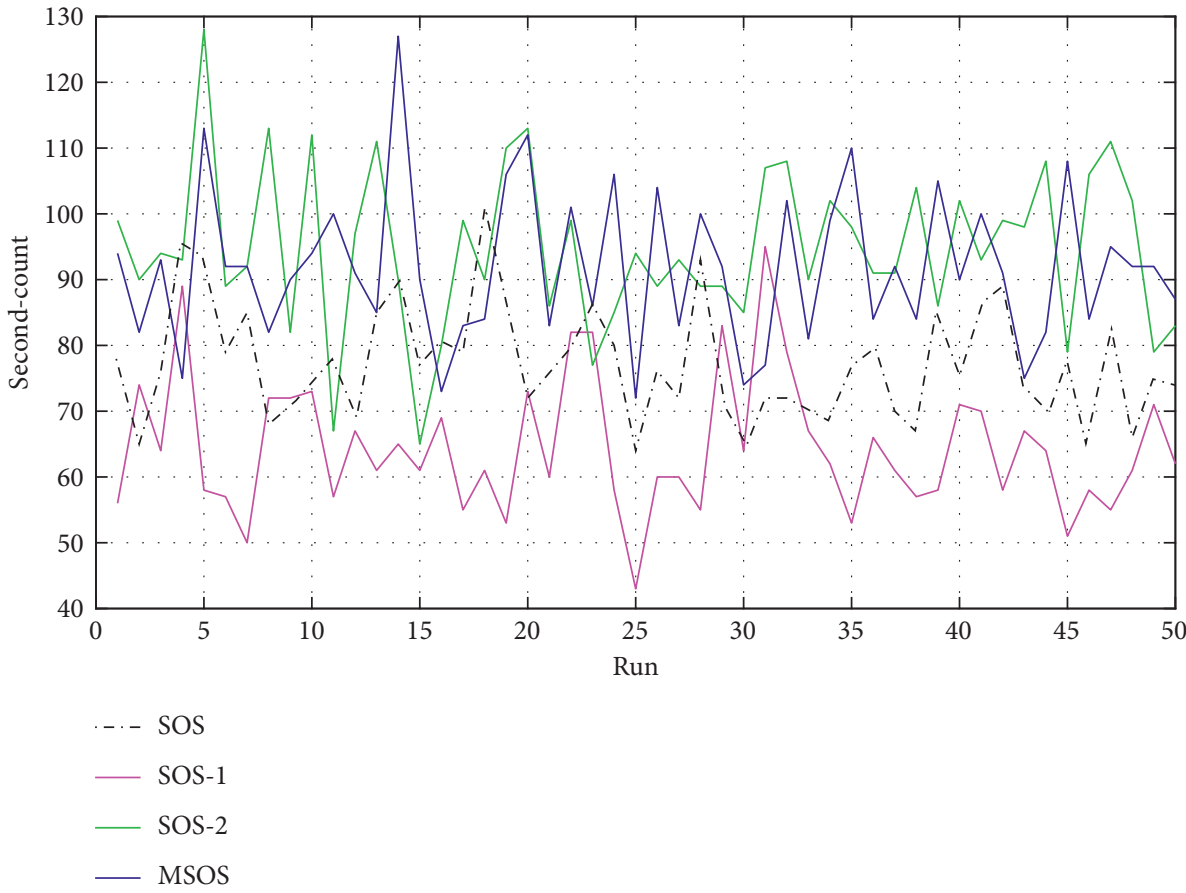

FIgURE 16: Number of times the new organism generated by the second modification technique is better than the current organism.

Table 13 also shows that the second modification technique has helped the parasitism strategy to create better organisms than that of the original SOS. Specifically, the number of times that the new organism was generated by the parasitism strategy is more than the current jth organism of SOS-2 and MSOS, which is 94.7400 and 91.7800 , respectively, whereas this number is only 77.3200 and 64.4000 for SOS and SOS-1, respectively. It is noted that the SOS and the
SOS- 1 do not use the second modification technique. The second-count value in each run of the four methods given in Figure 16 shows that in each run, the solution quality generated by the parasitic mechanism of the MSOS is better than that of the original parasitic mechanism.

Although all of SOS, SOS-1, SOS-2, and MSOS have determined the optimal configuration, the maximum, mean, and standard deviation of the fitness function of the MSOS 


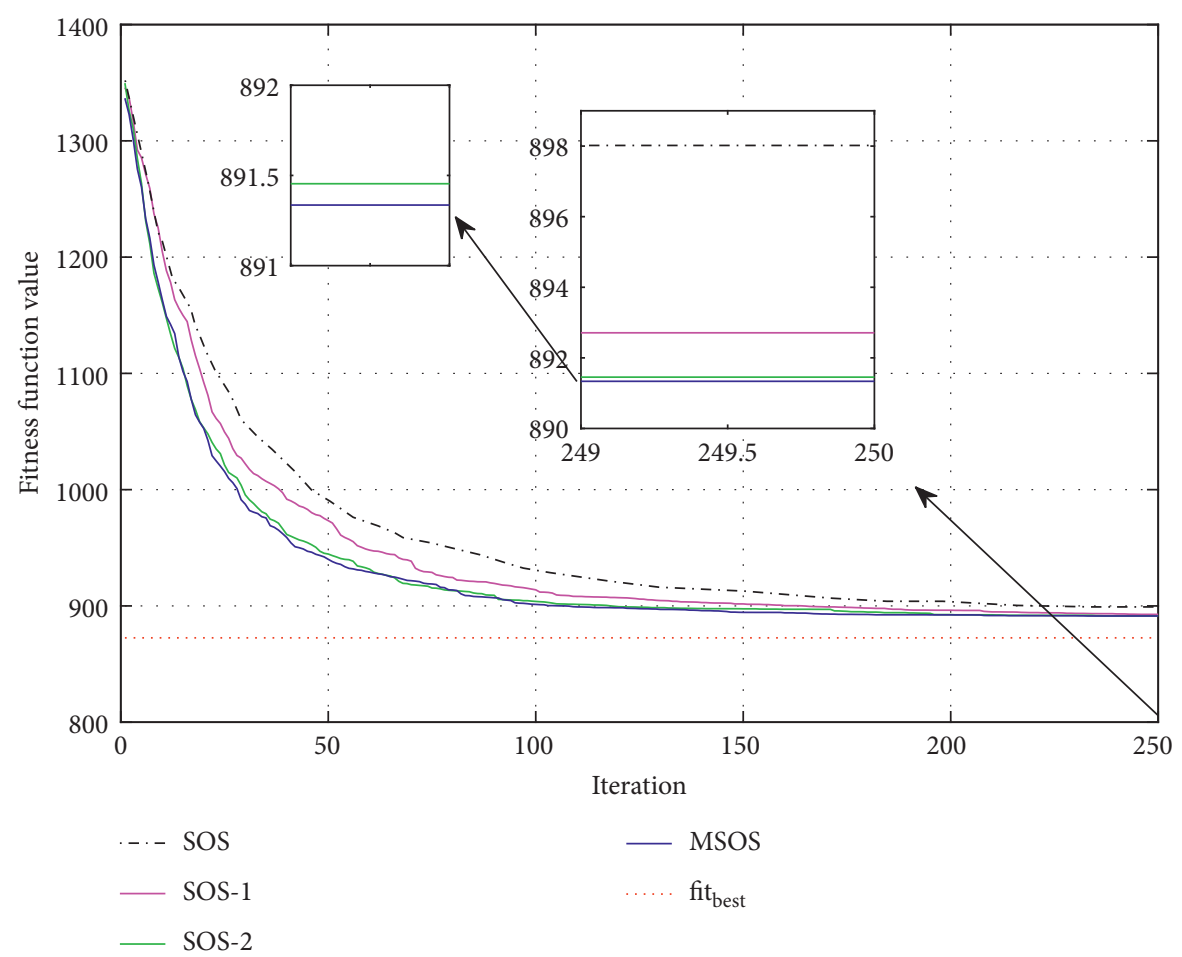

FIgURE 17: Mean convergence characters of the four methods for the 119-node system.

are the smallest and the success rate is the highest compared to those of the rest of the methods. Specifically, the mean value of the fitness functions of SOS, SOS-1, SOS-2, and MSOS is $898.0087,892.71,891.454$, and 891.3358 , respectively, with standard deviations of $17.904,12.0549,13.7885$, and 11.4839, respectively. The success rate of the MSOS is $24 \%$, which is the highest in all the four methods. In addition, the mean number of convergence iterations of the MSOS is also lower than that of the other methods. The mean convergence characteristics of SOS, SOS-1, SOS-2, and MSOS are shown in Figure 17, which shows that using two modification techniques makes the MSOS more efficient than the SOS. In addition, the second modification technique is more effective than the first one in improving the efficiency of the MSOS.

\section{Conclusion}

In this paper, the NR problem has been considered for power loss reduction base on the proposed MSOS. To improve the effectiveness of the MSOS, the so-far best solution has been updated as soon as some new solutions are created in the mutualism strategy to gain better information for the next strategy of the MSOS. In addition, the exploited search process in the parasitism strategy has been modified to create the solution that exists around the current solution. The modification of the parasitism strategy is implemented by adjusting the number of variables updated for the current solution. The comparison results between the MSOS and the SOS on different scale test distribution systems consisting of $14,33,69$, and 119 nodes indicate that the improvement level of the MSOS over the SOS is significant, with higher success rate for all the test systems. The success rate of the proposed MSOS method for the aforementioned systems has reached $100 \%, 88 \%, 70 \%$, and $20 \%$ respectively, whereas they have been only $94 \%, 76 \%, 46 \%$, and $10 \%$, respectively, for the SOS. Furthermore, the other indexes consisting of the average fitness value, STD, and the average convergence character in 50 runs of the MSOS are better than those of the SOS. Based on the compared results with the SOS and other the methods in the literature, the use of the proposed MSOS is a high contribution for finding more effective radial network configuration of minimizing power loss. In this work, the changes in load during the survey period have not been considered so that may be evaluated in the near future. Furthermore, the effectiveness of the MSOS may be evaluated for the different optimization problems in the power system field.

\section{Data Availability}

No data were used to support this study.

\section{Conflicts of Interest}

The authors declare no conflicts of interest.

\section{References}

[1] S. Gopiya Naik, D. K. Khatod, and M. P. Sharma, "Optimal allocation of combined DG and capacitor for real power loss minimization in distribution networks," International Journal of Electrical Power \& Energy Systems, vol. 53, pp. 967-973, 2013. 
[2] A. Merlin and H. Back, "Search for a minimal loss operating spanning tree configuration in an urban power distribution system," in Proceeding in 5th Power System Computation Conference (PSCC), pp. 1-18, Cambridge, UK, 1975.

[3] S. Civanlar, J. J. Grainger, H. Yin, and S. S. H. Lee, "Distribution feeder reconfiguration for loss reduction," IEEE Transactions on Power Delivery, vol. 3, no. 3, pp. 1217-1223, 1988.

[4] D. Shirmohammadi and H. W. Hong, "Reconfiguration of electric distribution networks for resistive line losses reduction," IEEE Transactions on Power Delivery, vol. 4, no. 2, pp. 1492-1498, 1989.

[5] J. Z. Zhu, "Optimal reconfiguration of electrical distribution network using the refined genetic algorithm," Electric Power Systems Research, vol. 62, no. 1, pp. 37-42, 2002.

[6] V. Ganesh, S. Sivanagaraju, and T. Ramana, "Feeder reconfiguration for loss reduction in unbalanced distribution system using genetic algorithm," International Journal of Electrical and Electronics Engineering, vol. 3, no. 12, pp. 754-762, 2009.

[7] P. Subburaj, K. Ramar, L. Ganesan, and P. Venkatesh, "Distribution system reconfiguration for loss reduction using genetic algorithm," Journal of Electrical Systems, vol. 2, no. 4, pp. 198-207, 2006.

[8] K. K. Kumar, N. Venkata, and S. Kamakshaiah, "FDR particle swarm algorithm for network reconfiguration of distribution systems," Journal of Theoretical and Applied Information Technology, vol. 36, no. 2, pp. 174-181, 2012.

[9] T. M. Khalil and A. V Gorpinich, "Reconfiguration for loss reduction of distribution systems using selective particle swarm optimization," International Journal of Multidisciplinary Sciences and Engineering, vol. 3, no. 6, pp. 16-21, 2012.

[10] A. Y. Abdelaziz, S. F. Mekhamer, F. M. Mohammed, and M. a L. Badr, "A modified particle swarm technique for distribution systems reconfiguration," The Online Journal on Electronics and Electrical engineering (OJEEE), vol. 1, no. 1, pp. 121-129, 2009.

[11] A. Saffar, R. Hooshmand, and A. Khodabakhshian, "A new fuzzy optimal reconfiguration of distribution systems for loss reduction and load balancing using ant colony search-based algorithm," Applied Soft Computing, vol. 11, no. 5, pp. 4021-4028, 2011.

[12] E. Carpaneto and G. Chicco, "Distribution system minimum loss reconfiguration in the hyper-cube ant colony optimization framework," Electric Power Systems Research, vol. 78, no. 12, pp. 2037-2045, 2008.

[13] A. Swarnkar, N. Gupta, and K. R. Niazi, "Adapted ant colony optimization for efficient reconfiguration of balanced and unbalanced distribution systems for loss minimization," Swarm and Evolutionary Computation, vol. 1, no. 3, pp. 129-137, 2011.

[14] T. T. Nguyen and T. T. Nguyen, "An improved cuckoo search algorithm for the problem of electric distribution network reconfiguration," Applied Soft Computing, vol. 84, Article ID 105720, 2019.

[15] T. T. Nguyen, A. V. Truong, and T. A. Phung, "A novel method based on adaptive cuckoo search for optimal network reconfiguration and distributed generation allocation in distribution network," International Journal of Electrical Power \& Energy Systems, vol. 78, pp. 801-815, 2016.

[16] A. Onlam, D. Yodphet, R. Chatthaworn, C. Surawanitkun, A. Siritaratiwat, and P. Khunkitti, "Power loss minimization and voltage stability improvement in electrical distribution system via network reconfiguration and distributed generation placement using novel adaptive shuffled frogs leaping algorithm," Energies, vol. 12, no. 3, p. 553, 2019.

[17] A. V. Truong, T. N. Ton, T. T. Nguyen, and T. L. Duong, "Two states for optimal position and capacity of distributed generators considering network reconfiguration for power loss minimization based on runner root algorithm," Energies, vol. 12, no. 1, p. 106, 2019.

[18] T. T. Nguyen, T. T. Nguyen, A. V. Truong, Q. T. Nguyen, and T. A. Phung, "Multi-objective electric distribution network reconfiguration solution using runner-root algorithm," Applied Soft Computing, vol. 52, pp. 93-108, 2017.

[19] S. H. Mirhoseini, S. M. Hosseini, M. Ghanbari, and M. Ahmadi, "A new improved adaptive imperialist competitive algorithm to solve the reconfiguration problem of distribution systems for loss reduction and voltage profile improvement," International Journal of Electrical Power \& Energy Systems, vol. 55, pp. 128-143, 2014.

[20] A. Mohamed Imran and M. Kowsalya, "A new power system reconfiguration scheme for power loss minimization and voltage profile enhancement using fireworks Algorithm," International Journal of Electrical Power \& Energy Systems, vol. 62, pp. 312-322, 2014.

[21] A. Jafari, H. Ganjeh Ganjehlou, F. Baghal Darbandi, B. Mohammadi-Ivatloo, and M. Abapour, "Dynamic and multi-objective reconfiguration of distribution network using a novel hybrid algorithm with parallel processing capability," Applied Soft Computing, vol. 90, Article ID 106146, 2020.

[22] X.-S. Yang, Engineering Optimization: An Introduction with Metaheuristic Applications, John Wiley \& Sons, Hoboken, NJ, USA, 2010.

[23] M.-Y. Cheng and D. Prayogo, "Symbiotic organisms search: a new metaheuristic optimization algorithm," Computers \& Structures, vol. 139, pp. 98-112, 2014.

[24] D. T. T. Do and J. Lee, "A modified symbiotic organisms search (mSOS) algorithm for optimization of pin-jointed structures," Applied Soft Computing, vol. 61, pp. 683-699, 2017.

[25] G. G. Tejani, V. J. Savsani, V. K. Patel, and S. Mirjalili, “Truss optimization with natural frequency bounds using improved symbiotic organisms search," Knowledge-Based Systems, vol. 143, pp. 162-178, 2018.

[26] D. Prayogo, M.-Y. Cheng, F. T. Wong, D. Tjandra, and D.-H. Tran, "Optimization model for construction project resource leveling using a novel modified symbiotic organisms search," Asian Journal of Civil Engineering, vol. 19, no. 5, pp. 625-638, 2018.

[27] V. F. Yu, A. A. N. P. Redi, C.-L. Yang, E. Ruskartina, and B. Santosa, "Symbiotic organisms search and two solution representations for solving the capacitated vehicle routing problem," Applied Soft Computing, vol. 52, pp. 657-672, 2017.

[28] Y. Wang, Y. W. Wu, and N. Xu, "Discrete symbiotic organism search with excellence coefficients and self-escape for traveling salesman problem," Computers \& Industrial Engineering, vol. 131, pp. 269-281, 2019.

[29] S. Saha and V. Mukherjee, "A novel chaos-integrated symbiotic organisms search algorithm for global optimization," Soft Computing, vol. 22, no. 11, pp. 3797-3816, 2018.

[30] S. Saha and V. Mukherjee, "A novel multi-objective modified symbiotic organisms search algorithm for optimal allocation of distributed generation in radial distribution system," Neural Computing and Applications, vol. 6, 2020.

[31] S. Saha and V. Mukherjee, "Optimal placement and sizing of DGs in RDS using chaos embedded SOS algorithm," IET 
Generation, Transmission \& Distribution, vol. 10, no. 14, pp. 3671-3680, 2016.

[32] T. P. Nguyen, V. N. Dieu, and P. Vasant, "Symbiotic organism search algorithm for optimal size and siting of distributed generators in distribution systems," International Journal of Energy Optimization and Engineering, vol. 6, no. 3, pp. 1-28, 2017.

[33] B. Das, V. Mukherjee, and D. Das, "DG placement in radial distribution network by symbiotic organisms search algorithm for real power loss minimization," Applied Soft Computing, vol. 49, p. 920, 2016.

[34] M. P. Lalitha, P.S. Babu, and B. Adivesh, "Optimal distributed generation and capacitor placement for loss minimization and voltage profile improvement using symbiotic organisms search algorithm," International Journal of Electrical Engineering, vol. 9, no. 3, pp. 249-261, 2016.

[35] M. Kenan Dosoglu, U. Guvenc, S. Duman, Y. Sonmez, and H. Tolga Kahraman, "Symbiotic organisms search optimization algorithm for economic/emission dispatch problem in power systems," Neural Computing and Applications, vol. 29, no. 3, pp. 721-737, 2018.

[36] D. C. Secui, "A modified symbiotic organisms search algorithm for large scale economic dispatch problem with valvepoint effects," Energy, vol. 113, pp. 366-384, 2016.

[37] T. L. Duong and T. T. Nguyen, "Network reconfiguration for an electric distribution system with distributed generators based on symbiotic organisms search," Engineering, Technology \& Applied Science Research, vol. 9, no. 6, pp. 49254932, 2019.

[38] A. E. Ezugwu and D. Prayogo, "Symbiotic Organisms Search Algorithm: theory, recent advances and applications," Expert Systems with Applications, vol. 119, pp. 184-209, 2019.

[39] A. Y. Abdelaziz, F. M. Mohamed, S. F. Mekhamer, and M. A. L. Badr, "Distribution system reconfiguration using a modified Tabu Search algorithm," Electric Power Systems Research, vol. 80, no. 8, pp. 943-953, 2010.

[40] M.-Y. Cheng and L.-C. Lien, "Hybrid artificial intelligencebased PBA for benchmark functions and facility layout design optimization," Journal of Computing in Civil Engineering, vol. 26, no. 5, pp. 612-624, 2012.

[41] M. E. Baran and F. F. Wu, "Network reconfiguration in distribution systems for loss reduction and load balancing," IEEE Transactions on Power Delivery, vol. 4, no. 2, pp. 14011407, 1989.

[42] R. Srinivasa Rao, S. V. L. Narasimham, M. Ramalinga Raju, and A. Srinivasa Rao, "Optimal network reconfiguration of large-scale distribution system using harmony search algorithm," IEEE Transactions on Power Systems, vol. 26, no. 3, pp. 1080-1088, 2011.

[43] H.-D. Chiang and R. Jean-Jumeau, "Optimal network reconfigurations in distribution systems II solution algorithms and numerical results," IEEE Transactions on Power Delivery, vol. 5, no. 3, pp. 1568-1574, 1990.

[44] A. Mohamed Imran, M. Kowsalya, and D. P. Kothari, “A novel integration technique for optimal network reconfiguration and distributed generation placement in power distribution networks," International Journal of Electrical Power \& Energy Systems, vol. 63, pp. 461-472, 2014.

[45] R. S. Rao, K. Ravindra, K. Satish, and S. V. L. Narasimham, "Power loss minimization in distribution system using network reconfiguration in the presence of distributed generation," IEEE Transactions on Power Systems, vol. 28, no. 1, pp. 317-325, 2013.
[46] N. T. Thuan, P. N. Hiep, T. V. Anh, P. A. Tuan, and N. T. Thang, "A backtracking search algorithm for distribution network reconfiguration problem," Lecture Notes in Electrical Engineering, Springer, Cham, Switzerland, pp. 223-230, 2015.

[47] D. Zhang, Z. Fu, and L. Zhang, "An improved TS algorithm for loss-minimum reconfiguration in large-scale distribution systems," Electric Power Systems Research, vol. 77, no. 5, pp. 685-694, 2007. 